\title{
Charge breeding of radioactive ions with EBIS and EBIT
}

\section{F. Wenander}

Keywords: Instrumentation for radioactive beams (fragmentation devices; fragment and isotope, separators incl. ISOL; isobar separators; ion and atom traps; weak-beam diagnostics; radioactivebeam ion sources); Ion sources (positive ions, negative ions, electron cyclotron resonance (ECR), electron beam (EBIS))

\begin{abstract}
Summary
A charge state breeder, which transforms externally injected singly charged ions to a higher charge state $\mathrm{q}^{+}$, is an important tool which has applications within atomic, nuclear and even particle physics. The charge breeding concept of radioactive ions has already been demonstrated at REXISOLDE/CERN with the use of an Electron beam Ion Source (EBIS) and at several facilities employing Electron Resonance Cyclotron Ion Sources (ECRIS). As will be demonstrated in this paper, EBIS and Electron Beam Ion Traps (EBIT), are well suited for the task as they are capable of delivering clean, highly charged beams within a short transformation time. The increasing demand for highly charged ions of all kind of elements and isotopes, stable and radioactive, to be used for low-energy experiments such as TITAN at TRIUMF and MATS at FAIR, but also for postacceleration to higher energies, is now pushing the development of the breeders. The next challenge will be to satisfy the needs, for example space-charge capacity, of the second generation radioactive beam facilities presently under construction or in the design stage, such as the MSU reaccelerator (ReA3), SPIRAL2, SPES and later on EURISOL. Radioactive trap facilities will also require high performance breeders geared towards rapid breeding times.

The requirements and the critical issues of the breeding concept will be discussed and a review of the different facilities, operational and planned, will be given. The paper does furthermore feature a summary of the extensive breeding experience gained under operational conditions at REXISOLDE, including results from dedicated beam cleaning tests, isotope production using intrap decay, high-current and continuous ion injection into the breeder, and closed-shell breeding.
\end{abstract}




\section{Charge breeding of radioactive ions with EBIS and EBIT}

\section{F. Wenander}

$C E R N, B E$ department,

1211 Geneva-23, Switzerland

E-mail: fredrik.wenander@cern.ch

ABSTRACT: A charge state breeder, which transforms externally injected singly charged ions to a higher charge state $\mathrm{q}+$, is an important tool which has applications within atomic, nuclear and even particle physics. The charge breeding concept of radioactive ions has already been demonstrated at REX-ISOLDE/CERN with the use of an Electron beam Ion Source (EBIS) and at several facilities employing Electron Resonance Cyclotron Ion Sources (ECRIS). As will be demonstrated in this paper, EBIS and Electron Beam Ion Traps (EBIT), are well suited for the task as they are capable of delivering clean, highly charged beams within a short transformation time. The increasing demand for highly charged ions of all kind of elements and isotopes, stable and radioactive, to be used for low-energy experiments such as TITAN at TRIUMF and MATS at FAIR, but also for postacceleration to higher energies, is now pushing the development of the breeders. The next challenge will be to satisfy the needs, for example space-charge capacity, of the second generation radioactive beam facilities presently under construction or in the design stage, such as the MSU re-accelerator (ReA3), SPIRAL2, SPES and later on EURISOL. Radioactive trap facilities will also require high performance breeders geared towards rapid breeding times.

The requirements and the critical issues of the breeding concept will be discussed and a review of the different facilities, operational and planned, will be given. The paper does furthermore feature a summary of the extensive breeding experience gained under operational conditions at REX-ISOLDE, including results from dedicated beam cleaning tests, isotope production using intrap decay, high-current and continuous ion injection into the breeder, and closed-shell breeding.

KEYWORDS: Instrumentation for radioactive beams (fragmentation devices; fragment and isotope, separators incl. ISOL; isobar separators; ion and atom traps; weak-beam diagnostics; radioactivebeam ion sources); Ion sources (positive ions, negative ions, electron cyclotron resonance (ECR), electron beam (EBIS)) 


\section{Contents}

1 Introduction 1

1.1 Field of application 1

1.2 Functioning of EBIS/T 2

1.3 Charge breeding of radioactive beams 3

2 The REXEBIS charge breeder 4

2.1 The setup 4

2.2 General results 6

2.3 Breeding efficiency 7

2.4 Hold-up time

2.5 Pulse structure 9

2.6 High current beams 10

2.7 Closed shell breeding 11

$\begin{array}{lll}2.8 & \text { Beam contamination and cleaning } & 14\end{array}$

$\begin{array}{lll}2.8 .1 & \text { Molecular beams } & 15\end{array}$

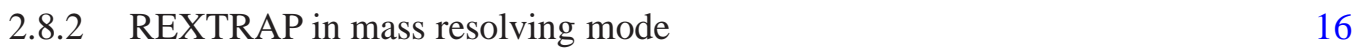

$\begin{array}{ll}2.9 \text { Continuous ion injection } & 17\end{array}$

2.10 In-trap decay 18

3 Other EBIB systems dedicated to post-acceleration or trap injection 19

$\begin{array}{lll}3.1 & \text { EBIB for post-acceleration } & 19\end{array}$

$\begin{array}{ll}3.2 & \text { EBIB for high-precision mass measurements }\end{array}$

4 Future RIB projects and upgrades $\quad 23$

5 Areas of improvement for EBIB 25

6 Conclusions and alternatives $\quad 27$

\section{Introduction}

\subsection{Field of application}

Highly charged stable ions at low energy have been requested by the atomic and surface physics communities for several decades, see for example [1-3]. High-precision mass measurements using traps $[4,5]$ benefit from the higher charge state as the resolution is given as:

$$
\frac{m}{\Delta m} \propto \frac{q \cdot B}{m} \cdot T_{R F} \sqrt{N}
$$


where $\mathrm{q}$ and $\mathrm{m}$ denote ion charge and mass, $\mathrm{B}$ the magnetic field, $\mathrm{T}_{R F}$ the duration of the radiofrequency excitation and $\mathrm{N}$ the number of measurement cycles. For short-lived radioactive ions the advantage becomes evident as the excitation time may be restricted by the half-life. Nuclear physics facilities now also make use of highly charged ions for acceleration up to and above the Coulomb barrier (i.e. a few $\mathrm{MeV} / \mathrm{u}$ ) for studying key reactions related to nucleosynthesis in astrophysics and the structure of nuclei far from stability. Even particle physics employs charge-bred stable ions, for instance gold, in the injectors for TeV-colliders [6] and ideas to use charge-bred radioactive ions for neutrino physics exist as well [7]. The advantage of charge breeding directly after the production stage is that a shorter and more power efficient post-accelerator can be employed as the attainable final energy is proportional to $\mathrm{q}$ and $\mathrm{q}^{2}$ for a linear accelerator (LINAC) and cyclotron, respectively. The externally injected singly charged ions undergo a breeding process to a q+ state to match the mass-to-charge limit of the accelerator. Contrary to the ion stripping case, where a gas jet or foils are employed, a pre-acceleration of single or double charged ions to some $100 \mathrm{keV} / \mathrm{u}$ is not required [8]. In principle, the charge breeding should be able to exhibit a higher transmission than traditional stripping, particularly for heavier elements where a two-stage stripping results in a few percent transmission in a single charge state.

Although charge breeding of stable elements was already carried out with CRYSIS [9] and DIONE [10] in the nineties, the important gain of momentum came in the last decade. The results from for example REXEBIS described below and the RHIC TestEBIS [11] (converting 1+ gold ions to at least $32+$ and providing $\mu$ s pulses of more than $10^{9}$ ions) among others, have promoted several new designs using EBIS/T as 1+ to q+ breeders, from here on denoted Electron Beam Ion Breeder (EBIB). Presently, the demand for a cost-efficient production of accelerated nuclear beams is pushing the development of two breeder options - EBIB and ECRIS. This paper will focus on charge breeding of radioactive ions as the growing field holds several upcoming Radioactive Ion Beam (RIB) facilities $[12,13]$. Examples of earlier reviews of EBIS/T and ECRIS as radioactive charge breeders are [14-17].

\subsection{Functioning of EBIS/T}

Atoms or ions that are introduced into an EBIS/T are ionized to higher charge states. If singly charge ions are injected over a lowered outer barrier, see figure 1 (top), the EBIS/T work as a breeder, but likewise neutral gas atoms or molecules can be introduced and the EBIS/T then acts as a source or trap of highly charged ions. Figure 1 (bottom) illustrates how a dense mono-energetic electron beam from an electron gun is used for the ionization process in an EBIS. The electron beam is focused and compressed by a strong magnetic field created by a surrounding solenoid. In most EBIS/T the electrons pass only once through the trapping region before they are absorbed at the electron collector. The ions inside the EBIS/T are confined radially by the electrostatic forces from the negatively charged electron beam and by the magnetic field, and longitudinally by potential barriers, established by cylindrical electrodes surrounding the beam. Inside the trapping region the high-energy electrons collide with ions. The ionization process is step-wise, removing one or a few electrons at each ionizing impact of the beam electrons, until the ions finally are extracted by lowering the outer barrier. Note that the ions are injected and extracted at the same side of the EBIS/T. By applying different voltages to the drift tubes the pulse shape of the extracted beam can be modulated. The ion motion inside the trap is a combination of radial oscillation in the 

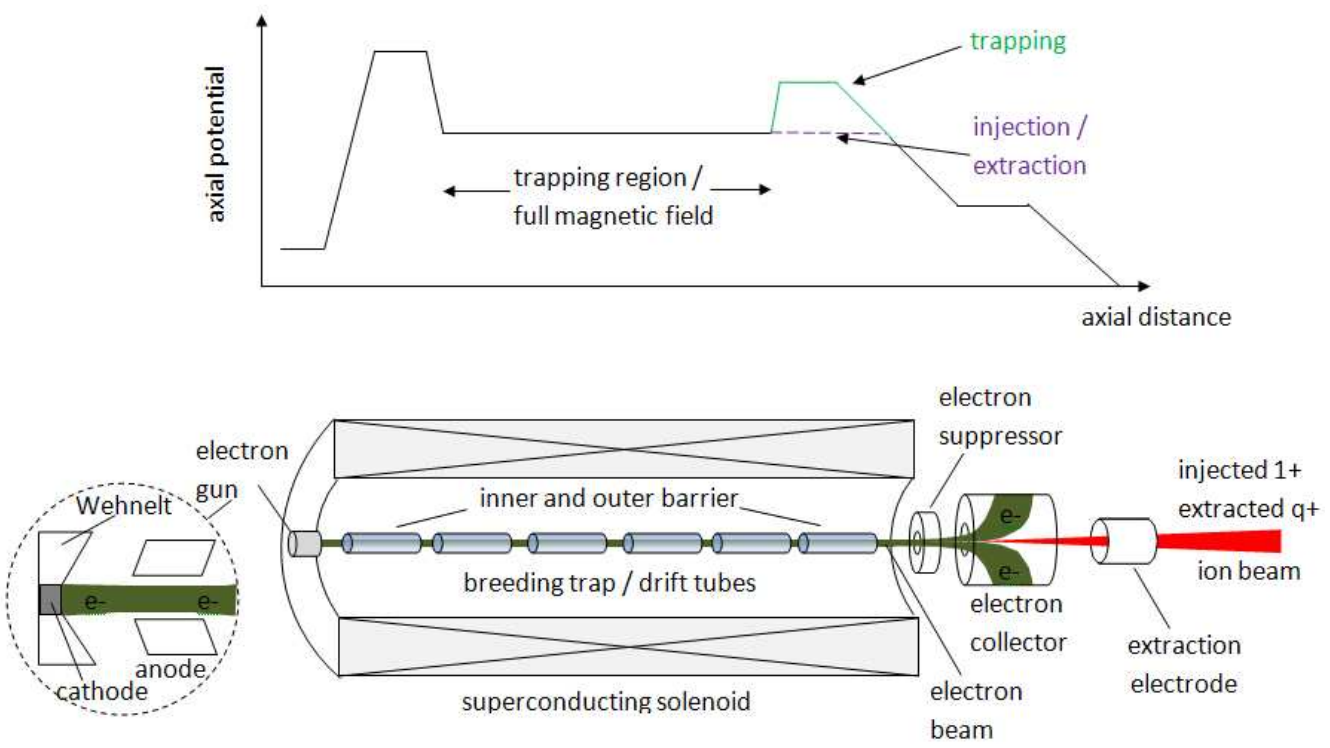

Figure 1. Top: axial potential inside an EBIS during pulsed ion injection, trapping and pulsed extraction. Bottom: key elements of an EBIS, including electron gun and collector, and an extended high magnetic field solenoid which compresses the electron beam in the trapping region in order to obtain a rapid ionization process.

electrostatic field of the electron beam with a superimposed azimuthal cyclotron motion around the magnetic field lines, and a relatively non-correlated bouncing between the end barriers.

The main characteristic entity describing an EBIS/T is the ionization factor, i.e. the product $\mathrm{j}_{e} \cdot \mathrm{T}_{\text {breeding }}$ of the electron-beam current-density $\mathrm{j}_{e}$ and the breeding time $\mathrm{T}_{\text {breeding. }}$. On average, to transform ions of charge state $\mathrm{q}$ to $\mathrm{q}+1$ an ionization factor $\mathrm{j}_{e} \cdot \mathrm{T}_{\text {breeding }}=\mathrm{e} / \sigma_{q \rightarrow q+1}$, where $\sigma_{q \rightarrow q+1}$ is the electron-impact ionization cross-section for charge state $\mathrm{q}$ to $\mathrm{q}+1$, is required. Thus, one can easily select a specific charge state by just varying the breeding time (see also figure 6), and there is no need for retuning other source parameters as in the case of an ECRIS. Nevertheless, also for an EBIS/T, the ions are statistically distributed over several charge states and therefore the inherent breeding efficiency is limited to between $20 \%$ and $40 \%$, depending on the $\mathrm{Z}$ of the ion. We shall later see that there exist tricks that can be used to overcome this limitation (section 2.7).

Traditionally an EBIS is characterized by an extended trapping region (typically $0.5 \mathrm{~m}$ or longer) surrounded by a solenoid, often superconducting with a field of a few $\mathrm{T}$, while the shorter magnetic field in an EBIT is created by a pair of strong Helmholtz coils. The space charge capacity of an EBIS is usually larger than for an EBIT, but because of the high electron current-density in the latter, it can produce very highly charged ions in a short time (even fully stripped uranium [18]).

\subsection{Charge breeding of radioactive beams}

The aspects of radioactive beam charge breeding are different from the stable ion breeding. Foremost, the rare ions have to be efficiently bred as a lack of delivered beam intensity may not simply be compensated by an increase of the injected 1+ beam. Consequently, the acceptance of the system - transverse and longitudinal - has to match the properties of the injected beam. Related 
to this is the necessity to minimize the radioactive contamination caused by losses in the system. The limited life-time of short-lived radioactive ions, down to some ms, stipulates a rapid charge breeding. A low superimposed ion contamination in the extracted beam is of particular importance for low-abundance, neutron-rich or deficient nuclei. The large number of isotopes in the nuclear landscape, over 3000, has a mass range from a few mass units to over 300, thereby demanding a large flexibility of the breeder system. The radioactive beam intensity spans an even larger range, from a few to over $10^{10}$ ions/s. Furthermore, crucial but not RIB specific criteria, are the space capacity (or ion throughput per time unit) and the most abundant charge state in the charge state distribution. The complexity of operation and the ease of changing charge state are also parameters to be considered as several beam changes (element, isotope or energy) may be requested per week, or even per day. The robustness of the system and the cost do also play a role. In the case of mass measurements by means of traps, the space charge inside the breeder is not a constraint as only a few ions are required to establish the cyclotron frequency. However, a low energy spread is desired, ideally only some $\mathrm{eV} / \mathrm{q}$, to reduce the effect on the mass resolution unless a dedicated cooling mechanism using, for instance, protons or electrons follows the breeder [19]. For mass resolution reasons, trap systems also benefit from the highest possible charge, while a post-accelerator in most cases only requires a mass-to-charge ratio (A/q) between 4 and 9 . A more extensive discussion of the criteria is found in [20].

The low-energetic, typically $30-60 \mathrm{keV}$, radioactive beams aimed for charge breeding are either produced in an Isotope Separator On-Line (ISOL) target-ion source [21], or in a Fragment Recoil Separator (FRS) connected to a thermalizing beam catcher [22], by interaction of driver beam particles with a target. In the ISOL situation the produced atoms or molecules effuse from the target to an ion source where ionization to $1+$, or rarely $2+$, takes place before the cocktail beam is accelerated and mass-separated. The quasi-continuous beams have emittances in the order of 15$30 \mathrm{~mm} \cdot \mathrm{mrad}(2 \sigma, 30 \mathrm{keV})$ [23]. From the beam catcher, singly charged fragmentation projectiles are extracted with approximately a factor 10 times smaller emittance. In order to reduce the transverse phase-space of the ISOL beams a gas-filled cooler of Radio Frequency Quadrupole (RFQ) or Penning trap type can be introduced and thereby enhance the injection efficiency into the breeder. Accumulation and bunching can also be performed if space charge allows and, bunch lengths of a few $\mu$ s are obtainable. A breeding scheme using an EBIB is shown in figure 2 with its main components. The system can indeed provide beams both for low-energy physics and post-acceleration. Succeeding the EBIB is an A/q-analyzer, with a resolution of $\sim 150$, to select the correct charge state and separate away residual gas ions produced in the breeder.

\section{The REXEBIS charge breeder}

\subsection{The setup}

The first dedicated charge breeding studies were performed at Stockholm [24] and Saclay [25] and served as a feasibility test for the REX-ISOLDE re-accelerator [26, 27] at CERN. The encouraging breeding efficiencies of $30 \%$ and $9.4 \%$ for $\mathrm{N}^{7+}$ and $\mathrm{Ar}^{14+}$ provided a solid basis for the construction of REXEBIS. REX-ISOLDE was the first $1+\rightarrow \mathrm{q}+$ based system to provide re-accelerated beams for nuclear physics experiments and have now supported a scientific program for many years [28]. 


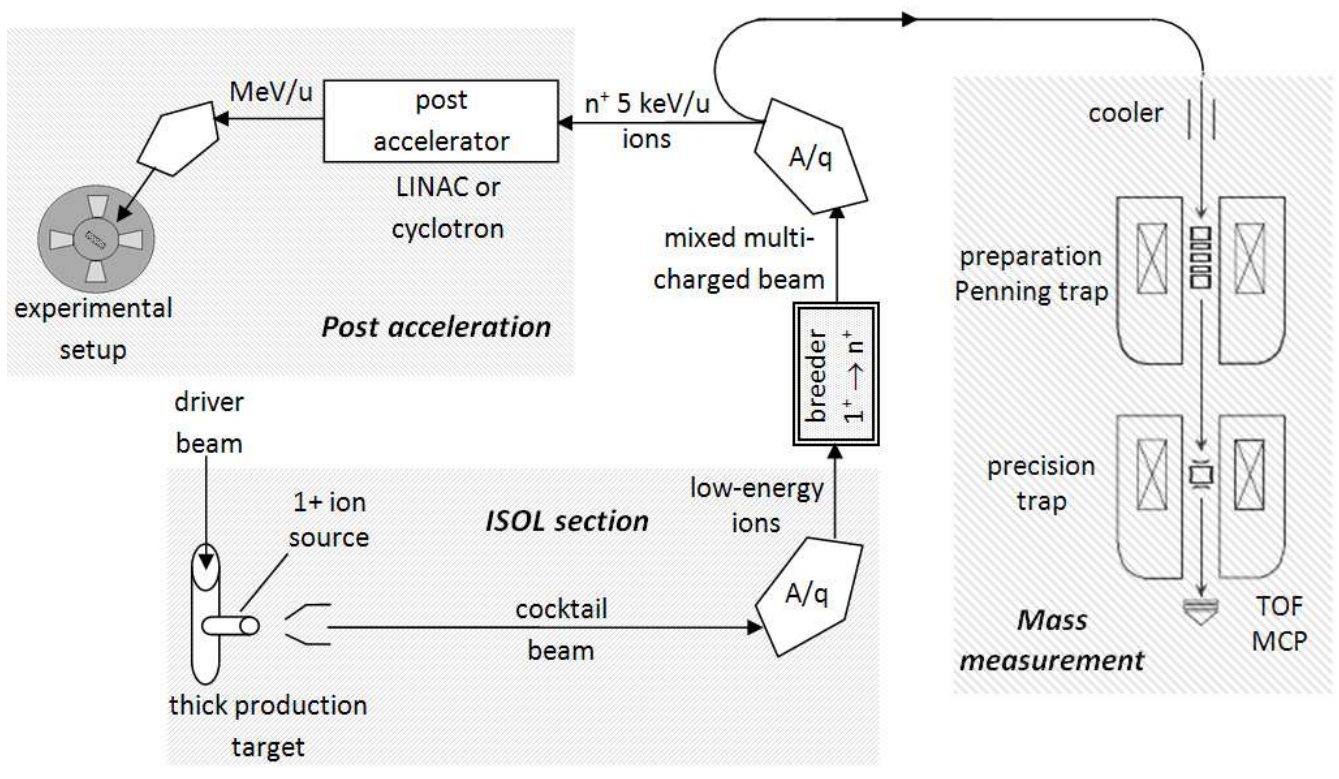

Figure 2. Schematic layout of a charge breeder system for an ISOL-beam based on an EBIB.

REX-ISOLDE makes use of a unique combination of a Penning trap (REXTRAP) and REXEBIS for the bunching and charge breeding of radioactive ions prior to their acceleration, see figure 3 . The 1+ ions exit the ISOLDE ion source as a quasi-continuous beam, with a time structure given by the proton impact on the primary target, typically with a few seconds interval and, the release time of the elements from the target, ranging from a few $10 \mathrm{~ms}$ to several minutes or longer. The beam, with a total energy of $30 \mathrm{keV}$, is mass-separated in one of two magnetic separators, before arriving to the gas-filled Penning trap, located at $30 \mathrm{kV}$. Due to the limited resolution of the separators $(\mathrm{R} \sim 1000$ and $\sim 5000)$ isobaric contaminations, stable or radioactive, may come with the desired radioactive ions. Accumulation and phase-space cooling takes place in the buffer-gas filled trap for as long time as the breeding process takes, that is several $10 \mathrm{~ms}$ in most cases, before the ions are transferred in a bunch to REXEBIS. For light ions a breeding time of only a few ms is required, nevertheless, the cooling time inside the trap has to be at least $10 \mathrm{~ms}$ to be effective. The EBIS efficiency is strongly dependent on the effectiveness of the phase-space cooling in the trap, optimized by tuning of the REXTRAP cooling cyclotron frequency. Inside the EBIS the ions are further ionized to an $\mathrm{A} / \mathrm{q}<4.5$, before being extracted at a variable potential to match the velocity acceptance $(5 \mathrm{keV} / \mathrm{u})$ of the RFQ cavity, the first element in the post-accelerator. The room temperature LINAC is pulsed with a duty cycle of $10 \%$ and provides beams to two different experimental stations, one of them being the Miniball gamma detector array [29]. The pulsed extraction from the EBIS is well matching the RF pulse length of the LINAC. For a self-extracted beam, that is ions leaving the trapping region without applying a potential gradient, a $\mathrm{T}_{F W H M}$ of approximately $30 \mu \mathrm{s}$ is attained. Between the EBIS and LINAC an achromatic A/q-separator selects the desired radioactive ions from ionized stable residual gas contaminations coming mainly from the trap and EBIS. REX-ISOLDE delivered its first beam to experiments in autumn 2001.

The REXEBIS itself can provide electron beam currents up to $460 \mathrm{~mA}$ with a current density of $\sim 200 \mathrm{~A} / \mathrm{cm}^{2}$, although it is mostly operated with around $200 \mathrm{~mA}$ and $100 \mathrm{~A} / \mathrm{cm}^{2}$. The electron 


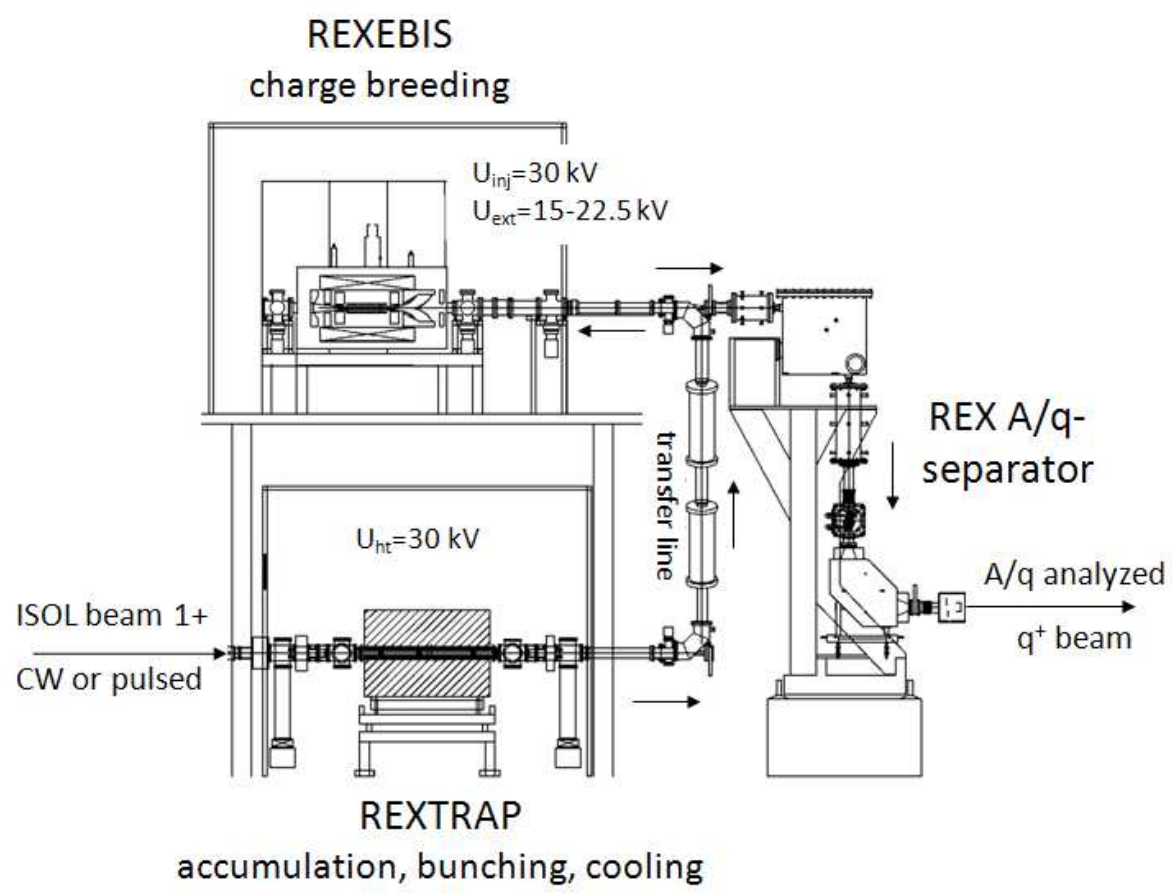

Figure 3. Layout of the REX-ISOLDE low energy part, including Penning trap, transfer line, breeder and A/q-separator.

beam is emitted from a $1.6 \mathrm{~mm}$ diameter $\mathrm{LaB}_{6}<310>$ crystal cathode, immersed in $0.2 \mathrm{~T}$ B-field. The room temperature trap with a length of $0.8 \mathrm{~m}$ is surrounded by a $2 \mathrm{~T}$ superconducting solenoid. A vacuum better than $10^{-10}$ mbar is achieved by a combination of high-compression molecular drag pumps and non-evaporable getters (NEG). The EBIS is situated on a high-voltage platform with a potential switchable between ion injection and extraction. The theoretical trapping capacity for a $5 \mathrm{keV}, 200 \mathrm{~mA}$ electron beam exceeds $2 \cdot 10^{10}$ charges, although the electron beam is never fully neutralized by the injected beam. Exhaustive information about REXTRAP and REXEBIS are found in [30, 31] and [20,32], respectively.

\subsection{General results}

Since the first beams of neutron-rich $\mathrm{Na}$ were delivered in fall 2001, numerous isotopes have been provided by REX-ISOLDE for nuclear astrophysics, nuclear structure physics and solid state physics at beam energies ranging from $300 \mathrm{keV} / \mathrm{u}$ up to $3.15 \mathrm{MeV} / \mathrm{u}$. By the end of 200972 radioactive isotopes of 25 different elements had been re-accelerated for physics, in addition to numerous stable isotopes for beam setup and tests. As demonstrated in figure 4, elements over the full mass range have been charge bred, the most challenging in terms of breeding being heavy neutron-rich ${ }^{224} \mathrm{Ra}$ and ${ }^{238} \mathrm{U}$. No surface physics processes affecting the charge bred ions take place in the bunching-cooling-breeding system of REX. However, charge exchange with the buffer gas atoms inside the Penning trap may occur if radioactive noble gases are to be charge bred [33]. For instance, neutron-rich ${ }^{91,92} \mathrm{Kr}$ isotopes suffered from a halved transmission through REXTRAP already at a cooling time of $50 \mathrm{~ms}$ due to recombination and, He-isotopes are problematic. Never- 
theless, it has been shown that the full nuclear chart can be covered, unless limited by short halflives. Even elements that are not extracted from ISOLDE may under favourable circumstances be delivered by making use of in-trap decay as explained below. Previous results from the coolingbunching-breeding system are presented in $[34,35]$.

\subsection{Breeding efficiency}

The transmission efficiency of the system has continually increased with gained operation experience. The total efficiency is defined as the particle current after the REX separator divided by the particle current injected into the trap, i.e. it encompasses trap transmission, breeding efficiency and REX separator transmission and selection. The Penning trap efficiency ranges from $20 \%$ to close to $60 \%$, the lower values occurring for elements with $\mathrm{A}<15$. Light elements are reflected at the injection side and have a large energy spread inside the trap making it difficult to confine them [30]. In addition, the cooling process is less efficient for the very light elements as the mass difference between the buffer-gas and the injected ions is relatively large. The total low-energy efficiency for a selection of beams, both stable pilot beams and radioactive, is plotted in figure 4 (top) with complementing data listed in table 1 . The selection shows cases with good performance to reflect the potential of the machine, as the excluded lower efficiency cases are due to a non-optimized setup. An overall efficiency of at least $3 \%$ can be expected for the full mass spectrum, with record efficiencies in the $20 \%$ region. As mentioned the light elements are problematic due to insufficient cooling in the Penning trap, but also heavier elements show a lower transmission. The reason for a lower heavy-ion efficiency is partly due to a broader charge state distribution than for low-Z elements. Secondly, a poor vacuum $\left(\sim 2 \cdot 10^{-7} \mathrm{mbar}\right)$ in the REX mass separator, which has a length of about $6 \mathrm{~m}$ until the focal point after the separator magnet, causes charge exchange losses of the highly charged ions. Using Müller-Salzborn's formula for charge exchange [36] and assuming a $13 \mathrm{eV}$ ionization potential of the residual gas, the separator losses for ions with charge $40+$ can be estimated to $25 \%$ and, approximately $5 \%$ for charges around $10+$. A semi-empirical evaluation of the efficiency for the REX low-energy stage is given in table 2.

\subsection{Hold-up time}

The hold-up time denotes the preparation time of the ISOL-ions before they are post-accelerated. The total hold-up time when the REX low-energy system is operated in normal mode is comprised of the cooling time in REXTRAP and the consecutive breeding time inside REXEBIS. For synchronization reasons the former has in general the same length as the latter, unless the breeding time is shorter than $10 \mathrm{~ms}$, then the cooling time still has to be between 10 and $20 \mathrm{~ms}$ in order to fully cool the ions. That means a $50 \mathrm{~Hz}$ repetition rate is used for masses below 40 . In figure 4 (middle) the EBIS breeding time is plotted for the same elements as given in the efficiency plot. The mass-to-charge ratio, plotted in figure 4 (bottom), varies between 2.5 and 4.5 and the specific value for each case is chosen so over-lapping A/q-peaks of contaminant ions are avoided. For the heavier elements the breeding time can be as long as $300 \mathrm{~ms}$, resulting in a total hold-up time of $600 \mathrm{~ms}$. In order to maintain a sufficiently high repetition rate the chosen breeding time might not be optimum for the delivered charge state, as in the case of radon for example, but slightly shorter. The hold-up time is comparable with or shorter than the diffusion-effusion time of the ISOLDE target-ion source for most elements, but very short-lived elements, such as ${ }^{11} \mathrm{Li}\left(\mathrm{t}_{1 / 2}=8.5 \mathrm{~ms}\right)$, are 


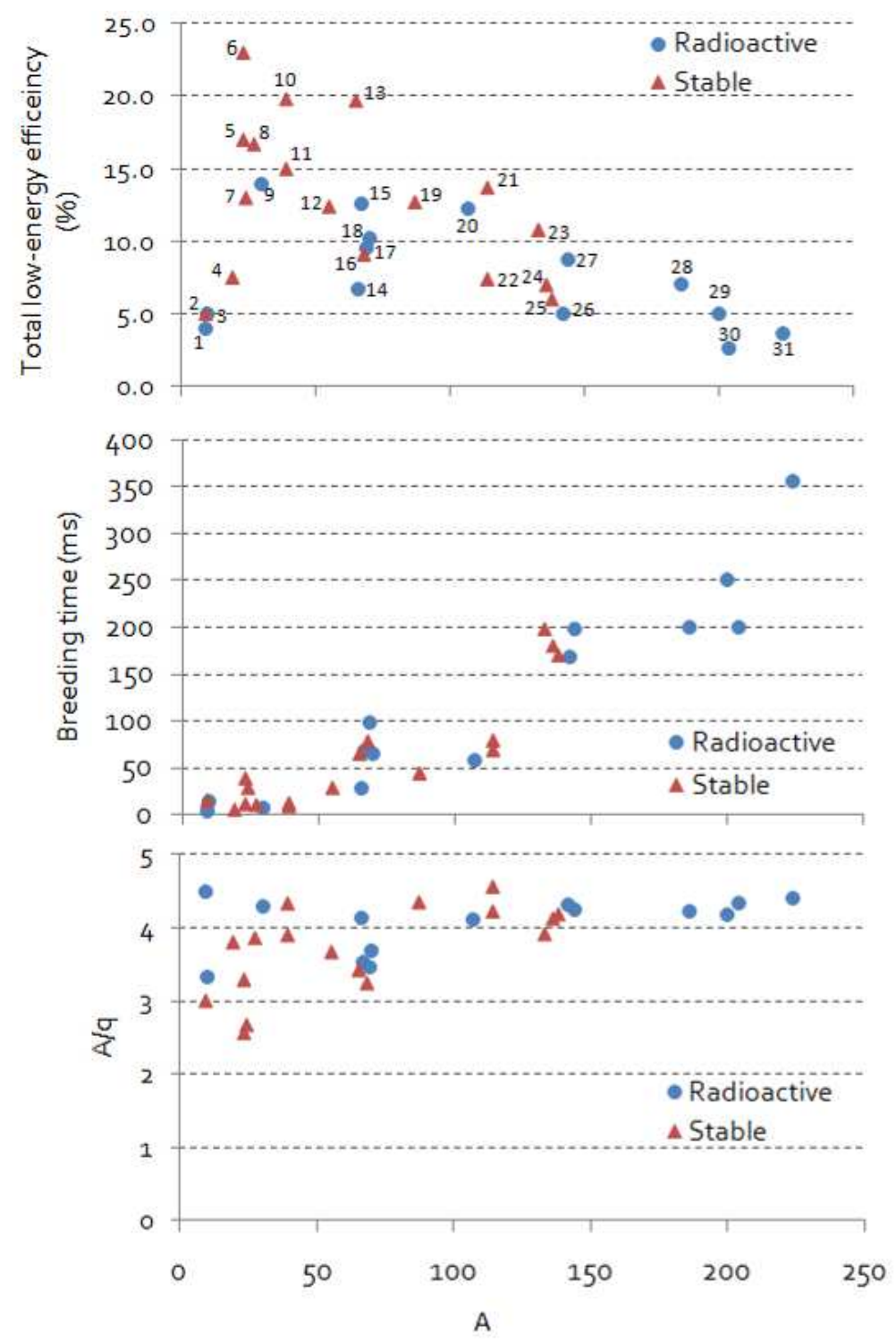

Figure 4. Top: measured total low-energy efficiency (trap + EBIS + REX mass separator) for a selection of elements, details listed in table 1. Middle: charge breeding time for the same elements (excluding coolingbunching time inside REXTRAP). Bottom: mass-to-charge ratio for the same elements.

difficult to deliver without significant decay losses. The use of continuous injection into the EBIS described below can curb the hold-up time. Closed shell breeding, as exemplified with ${ }^{23} \mathrm{Na}^{9+}$ in this plot, leads on the other hand to longer breeding times (in the sodium case to $38.5 \mathrm{~ms}$ ).

The breeding time is dependent on the electron beam settings, ion mass, the desired $\mathrm{A} / \mathrm{q}$ and the ion beam tuning. The theoretical electron current density has been confirmed with breeding tests. For instance, injected ${ }^{138} \mathrm{Xe}$ is charge bred to $32+/ 33+$ within $180 \mathrm{~ms}$ with a $190 \mathrm{~mA}$ current, corresponding to a current density of approximately $110 \mathrm{~A} / \mathrm{cm}^{2}$. Nevertheless, for incorrectly injected ions with only a temporary overlap with the electron beam, a breeding time of $330 \mathrm{~ms}$ was needed to reach $31+/ 32+$ [37], translating into an effective electron current density of $60 \mathrm{~A} / \mathrm{cm}^{2}$. 
Table 1. Cooling and breeding data for elements presented in figure 4.

\begin{tabular}{|c|c|c|c|c|c|c|c|}
\hline & Element & $\mathbf{A} / \mathbf{q}$ & $\mathbf{T}_{1 / 2}$ & $\begin{array}{c}\mathbf{T}_{\text {period }} \\
(\mathbf{m S})\end{array}$ & $\begin{array}{c}\mathbf{T}_{\text {breeding }} \\
\text { (ms) }\end{array}$ & $\begin{array}{c}\text { Efficiency } \\
(\%)\end{array}$ & Comments \\
\hline 1 & ${ }^{9} \mathrm{Li}^{2+}$ & 4.5 & $178.3 \mathrm{~ms}$ & 20 & 4 & 4 & \\
\hline 2 & ${ }^{9} \mathrm{Be}^{3+}$ & 3 & stable & 20 & 5 & 5 & \\
\hline 3 & ${ }^{10} \mathrm{Be}^{3+}$ & 3.333 & $1.6 \mathrm{My}$ & $20 ?$ & 15 & 5 & ${ }^{22} \mathrm{Ne}$ buffer gas, $\mathrm{T}_{\text {period }}$ uncertain \\
\hline 4 & ${ }^{19} \mathrm{~F}^{5+}$ & 3.8 & stable & 20 & 5 & 7.5 & \\
\hline 5 & ${ }^{23} \mathrm{Na}^{7+}$ & 3.286 & stable & 20 & 11 & 17 & \\
\hline 6 & ${ }^{23} \mathrm{Na}^{9+}$ & 2.556 & stable & 40 & 38.5 & 23 & closed shell breeding \\
\hline 7 & ${ }^{24} \mathrm{Mg}^{9+}$ & 2.667 & stable & 30 & 28.5 & 13 & \\
\hline 8 & ${ }^{27} \mathrm{Al}^{7+}$ & 3.857 & stable & 20 & 10 & 16.7 & $\mathrm{AlF}^{+}$molecule injected \\
\hline 9 & ${ }^{30} \mathrm{Mg}^{7+}$ & 4.286 & $335 \mathrm{~ms}$ & 20 & 8 & 14 & large error bars \\
\hline 10 & ${ }^{39} \mathrm{~K}^{9+}$ & 4.333 & stable & 20 & 9 & 19.8 & closed shell breeding \\
\hline 11 & ${ }^{39} \mathrm{~K}^{10+}$ & 3.9 & stable & 20 & 12 & 15 & \\
\hline 12 & ${ }^{55} \mathrm{Mn}^{15+}$ & 3.667 & stable & 30 & 28.5 & 12.4 & \\
\hline 13 & ${ }^{65} \mathrm{Cu}^{19+}$ & 3.421 & stable & 100 & 65 & 19.7 & closed shell breeding \\
\hline 14 & ${ }^{66} \mathrm{Ni}^{16+}$ & 4.125 & $54.6 \mathrm{~h}$ & 30 & 28.5 & 6.7 & \\
\hline 15 & ${ }^{67} \mathrm{Cu}^{19+}$ & 3.526 & $61.9 \mathrm{~h}$ & 100 & 68 & 12.6 & closed shell breeding \\
\hline 16 & ${ }^{68} \mathrm{Zn}^{21+}$ & 3.238 & stable & 80 & 78 & 9.1 & \\
\hline 17 & ${ }^{69} \mathrm{Cu}^{20+}$ & 3.45 & $3 \mathrm{~m}$ & 100 & 98 & 9.5 & \\
\hline 18 & ${ }^{70} \mathrm{Cu}^{19+}$ & 3.684 & $44.5 \mathrm{~s}$ & 70 & 65 & 10.2 & single isomer \\
\hline 19 & ${ }^{87} \mathrm{Rb}^{20+}$ & 4.35 & stable & 50 & 44 & 12.7 & \\
\hline 20 & ${ }^{107} \mathrm{Sn}^{26+}$ & 4.115 & $4.11 \mathrm{~h}$ & 82 & 59 & 12.2 & \\
\hline 21 & ${ }^{114} \mathrm{Cd}^{25+}$ & 4.56 & stable & 70 & 69 & 13.7 & \\
\hline 22 & ${ }^{114} \mathrm{Sn}^{27+}$ & 4.222 & stable & 82 & 79 & 7.4 & \\
\hline 23 & ${ }^{133} \mathrm{Cs}^{34+}$ & 3.912 & stable & 200 & 198 & 10.8 & \\
\hline 24 & ${ }^{136} \mathrm{Xe}^{33+}$ & 4.121 & stable & 183 & 180 & 7 & large error bars \\
\hline 25 & ${ }^{138} \mathrm{Ba}^{33+}$ & 4.182 & stable & 173 & 170 & 6 & $\mathrm{BaF}^{+}$molecule injected \\
\hline 26 & ${ }^{142} \mathrm{Ba}^{33+}$ & 4.303 & $10.7 \mathrm{~m}$ & 173 & 168 & 5 & $\mathrm{BaF}^{+}$molecule injected \\
\hline 27 & ${ }^{144} \mathrm{Xe}^{34+}$ & 4.235 & $1.15 \mathrm{~s}$ & 200 & 198 & 8.7 & \\
\hline 28 & ${ }^{186} \mathrm{Hg}^{44+}$ & 4.227 & $1.4 \mathrm{~m}$ & 204 & 200 & 7.1 & \\
\hline 29 & ${ }^{200} \mathrm{Po}^{48+}$ & 4.167 & $12.5 \mathrm{~h}$ & 255 & 250 & 5 & \\
\hline 30 & ${ }^{204} \mathrm{Rn}^{47+}$ & 4.34 & $1.24 \mathrm{~m}$ & 204 & 200 & 2.6 & non-optimal breeding time \\
\hline 31 & ${ }^{224} \mathrm{Ra}^{51+}$ & 4.392 & $3.66 \mathrm{~d}$ & 357 & 355 & 3.7 & \\
\hline
\end{tabular}

\subsection{Pulse structure}

The pulsed extraction from REXEBIS leads to an improved signal-to-noise ratio at the experimental station for low intensity beams. With a breeding time of $50 \mathrm{~ms}$ and $200 \mu$ s measurement time per pulse, $24 \mathrm{~h}$ of beam time corresponds to approximately 15 minutes of data collection. On 
Table 2. Estimated Penning trap transmission, calculated single charge-state occupancy after breeding, calculated charge-exchange losses in REX mass separator and theoretical attainable efficiency for REX lowenergy stage for different masses under present conditions.

\begin{tabular}{|c|c|c|c|c|c|}
\hline A & $\mathbf{q}$ & $\begin{array}{l}\text { Estimated } \\
\text { Penning trap } \\
\text { efficiency \% }\end{array}$ & $\begin{array}{l}\text { Single charge state } \\
\text { occupation \% } \\
\text { (no closed shell } \\
\text { breeding) }\end{array}$ & $\begin{array}{l}\text { Charge exchange losses } \\
\text { in REX separator \% } \\
\text { (present separator } \\
\text { vacuum) }\end{array}$ & $\begin{array}{l}\text { Attainable low } \\
\text { energy } \\
\text { efficiency \% }\end{array}$ \\
\hline 20 & $5+$ & 35 & 42 & 2.3 & 14 \\
\hline 56 & $13+$ & 55 & 30 & 7 & 15 \\
\hline 96 & $23+$ & 60 & 26 & 21 & 13 \\
\hline 137 & $33+$ & 60 & 25 & 32 & 9 \\
\hline 197 & $46+$ & 55 & 23 & 38 & 7 \\
\hline 238 & $54+$ & 50 & 23 & & 14 \\
\hline
\end{tabular}

the other hand, the instantaneous particle rate will be very high for high intensity beams. An average beam intensity of $10^{5}$ particles per second (pps) results for the same timing parameters in an instantaneous rate of $2.5 \cdot 10^{7}$ pps in the pulse, which may lead to missed events in the DAQ or pile-up problems in the detector system. Hence, slow ion extraction is commonly requested by the experiments. The ions are then extracted during some hundred $\mu \mathrm{s}$, the upper limit of $800 \mu \mathrm{s}$ being the length of the LINAC RF pulse, by slowly lowering the outer trapping barrier [38]. The optimal EBIS trap settings are dependent on the ion mass and charge, therefore time-of-flight diagnostics of the extracted beam is mandatory during the setting-up phase. As the ionization efficiency can be maintained, this flexibility allows the beam pulse length to be tailored to the experiment. Figure 5 shows the time structure, for self-extraction and slow extraction, of ${ }^{224} \mathrm{Ra}^{52+}$ measured with a particle detector at the Miniball setup.

\subsection{High current beams}

The maximal current throughput is not primarily limited by the REXEBIS storage capacity, but by space-charge effects inside REXTRAP. Apart from an altered cyclotron resonance frequency at large trap loads [39], which can be compensated for, the space-charge inside the Penning trap leads to insufficient phase-space cooling [31] and thereby less efficient ion injection into the EBIS. Alternatives to the sideband cooling scheme have been tried without real success [40]. Nonetheless, numerous tests with stable beams, for example $\mathrm{Li}, \mathrm{Na}$ and $\mathrm{K}$, have demonstrated that the REX lowenergy system is capable of handling most beam intensities provided by ISOLDE [41].

For instance, different intensities of a ${ }^{23} \mathrm{Na}+$ beam were injected into the trap while the current of the charge bred ${ }^{23} \mathrm{Na}^{7+}$ was recorded after the REX mass separator. The efficiency of the lowenergy section is plotted as function of injected current in figure 6. At the test a modest electron beam of $176 \mathrm{~mA}$ was used for a period time of $20 \mathrm{~ms}$ and the optimized breeding time was found to vary between 11 and $13 \mathrm{~ms}$. The REXTRAP cyclotron frequency was fine-tuned for the various intensities. A drop in efficiency from $14 \%$ for low currents to $5 \%$ for $2.5 \mathrm{nA}$ injected been was 

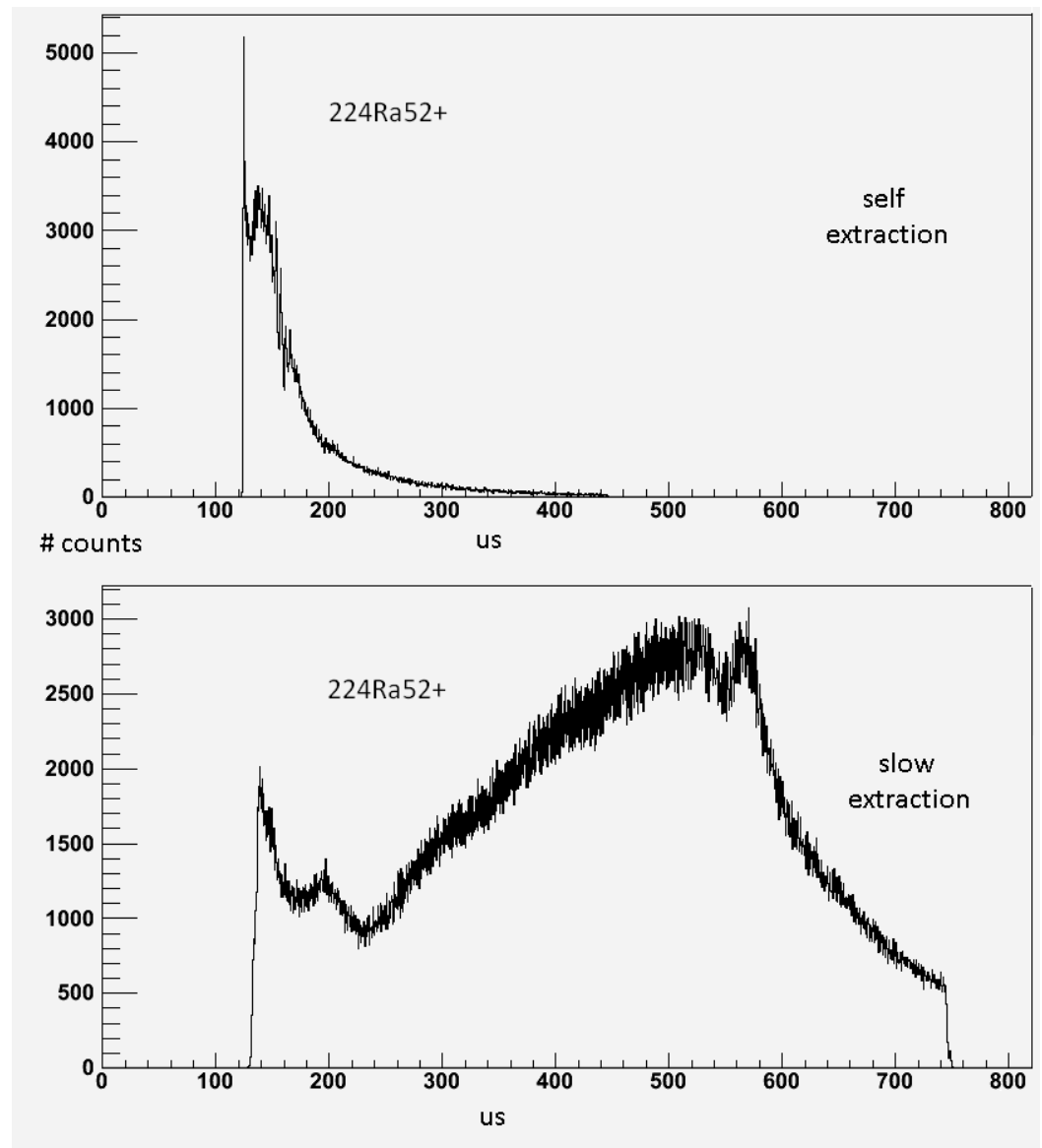

Figure 5. Time structure for self- and slow extraction from REXEBIS. The beam particles, ${ }^{224} \mathrm{Ra}^{52+}$, were measured with a particle detector at the Miniball setup. The number of counts in the two peaks is different due to varying beam current. Note that the tail of the slow extraction peak is cut due to the LINAC RF being stopped at 750 us.

recorded and attributed to the worsened emittance of the ion beam extracted from the trap. The highest current is equivalent to $3 \cdot 10^{8}$ ions/pulse for a repetition rate of $50 \mathrm{~Hz}$, thus far below the space-charge capacity $(<10 \%)$ of the EBIS. A high-intensity case of radioactive ${ }^{108} \mathrm{Sn}$ confirmed the measurement as an efficiency of $>7 \%$ could be obtained for injected currents of $100 \mathrm{pA}$ and a cycle time of $70 \mathrm{~ms}$ [37]. The use of continuous injection directly into REXEBIS may allow for higher beam intensities as will be discussed below.

\subsection{Closed shell breeding}

It is well known that the electron beam energy in an EBIS can be used to maximize the abundance of a particular charge state by taking advantage of ionization thresholds and recombination resonances $[42,43]$. By adjusting the electron beam energy accurately just below the ionization potential of an electron shell closure, a large fraction of the ions can end up in a single charge state. The method makes use of the large gap in ionization energy at the shell closure of atomic ions. The radiative recombination (RR) counteracts the electron impact ionization and the steady state 


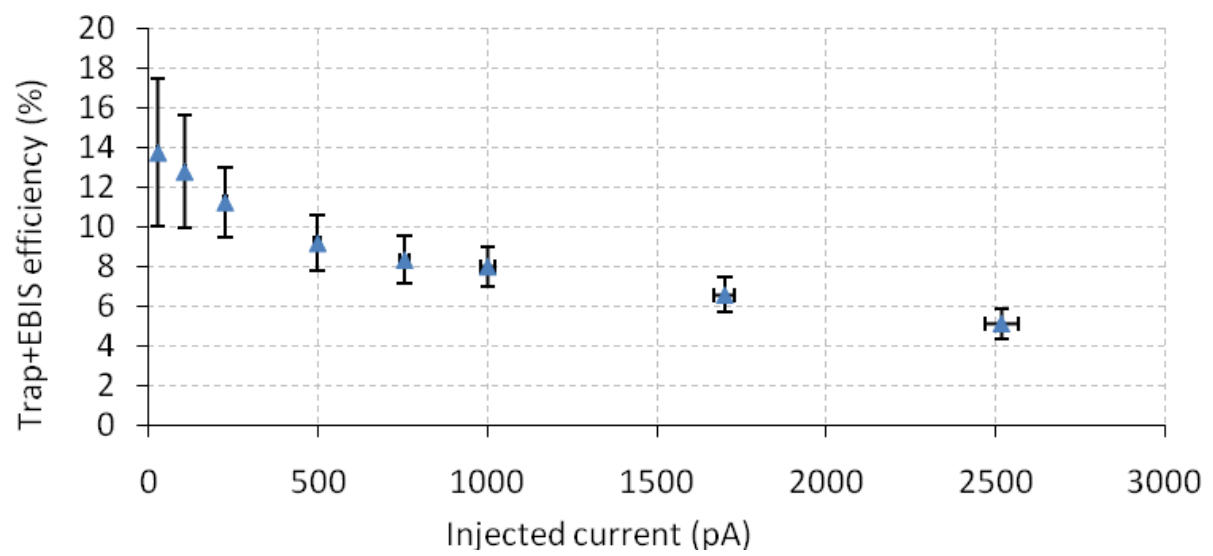

Figure 6. Total low-energy transmission efficiency as function of injected ${ }^{23} \mathrm{Na}^{+}$current into REXTRAP.

abundance of the highest charge states for long breeding times is determined by the ratio of the ionization cross-section and the RR cross-section.

The closed shell breeding has been tested at the REX-ISOLDE setup. For instance, a beam of stable potassium has been used. A large ionization potential gap occurs between $\mathrm{K}^{16+} \rightarrow \mathrm{K}^{17+}$ $(1055 \mathrm{eV})$ and the first K-shell ionization $\mathrm{K}^{17+} \rightarrow \mathrm{K}^{18+}(4423 \mathrm{eV})$, which is compatible with the REXEBIS electron beam energy of 3500 to $5500 \mathrm{eV}$. The electron beam energy was adjusted to approximately $4375 \mathrm{eV}$, with an energy distribution of $\pm 25 \mathrm{eV}$ caused by the potential depth of the electron beam itself. The choice of beam energy meant that the breeder could be operated with its normal settings, i.e. with a beam current of $300 \mathrm{~mA}$ and a current density evaluated to $130 \mathrm{~A} / \mathrm{cm}^{2}$. In addition, by choosing a high electron beam energy close to the ionization potential for $\mathrm{K}^{17+}$ to $\mathrm{K}^{18+}$, the ratio of ionization to RR rates was optimized. The $1+$ beam was first bunched and cooled in REXTRAP and thereafter injected into the EBIS for breeding, following the standard concept. The breeding times were varied from $20 \mathrm{~ms}$ to $250 \mathrm{~ms}$, while the REXTRAP cooling time was fixed to $250 \mathrm{~ms}$ to avoid the influence of varying space-charge effects. The setup had a satisfactory efficiency, but was not fully optimized.

In order to estimate the influence of RR and charge exchange effects, CBSIM [44] simulations were performed using the experimental beam energy but not including ion heating. The simulation results shown in figure 7 suggested a major occupancy in the $\mathrm{K}^{17+}$ charge state, approximately $70 \%$ after $250 \mathrm{~ms}$ breeding time. The influence of RR for a $4375 \mathrm{eV}$ electron beam energy is negligible, as well as charge exchange effects even at a pressure of $10^{-9}$ mbar (one order of magnitude higher than expected), as displayed in the two panels.

The experimental results revealed a different charge state distribution, as shown in figure 8 . The combined transmission of REXTRAP and REXEBIS for different charges states is plotted as function of the breeding time. The transmission curves reflect the charge state distribution inside the EBIS at the moment of extraction. One finds that even for $250 \mathrm{~ms}$ breeding time the 16+ charge state dominates, with $40 \%$ more ions than $17+$. The interpolating line for $17+$ predicts a charge state dominance for breeding times exceeding $\sim 350 \mathrm{~ms}$. Measurement limitations prevented investigation of such long breeding times. The reason for a lower peak charge state than expected could be ion heating effects resulting in a lower effective electron current density leading to a longer breeding time. 


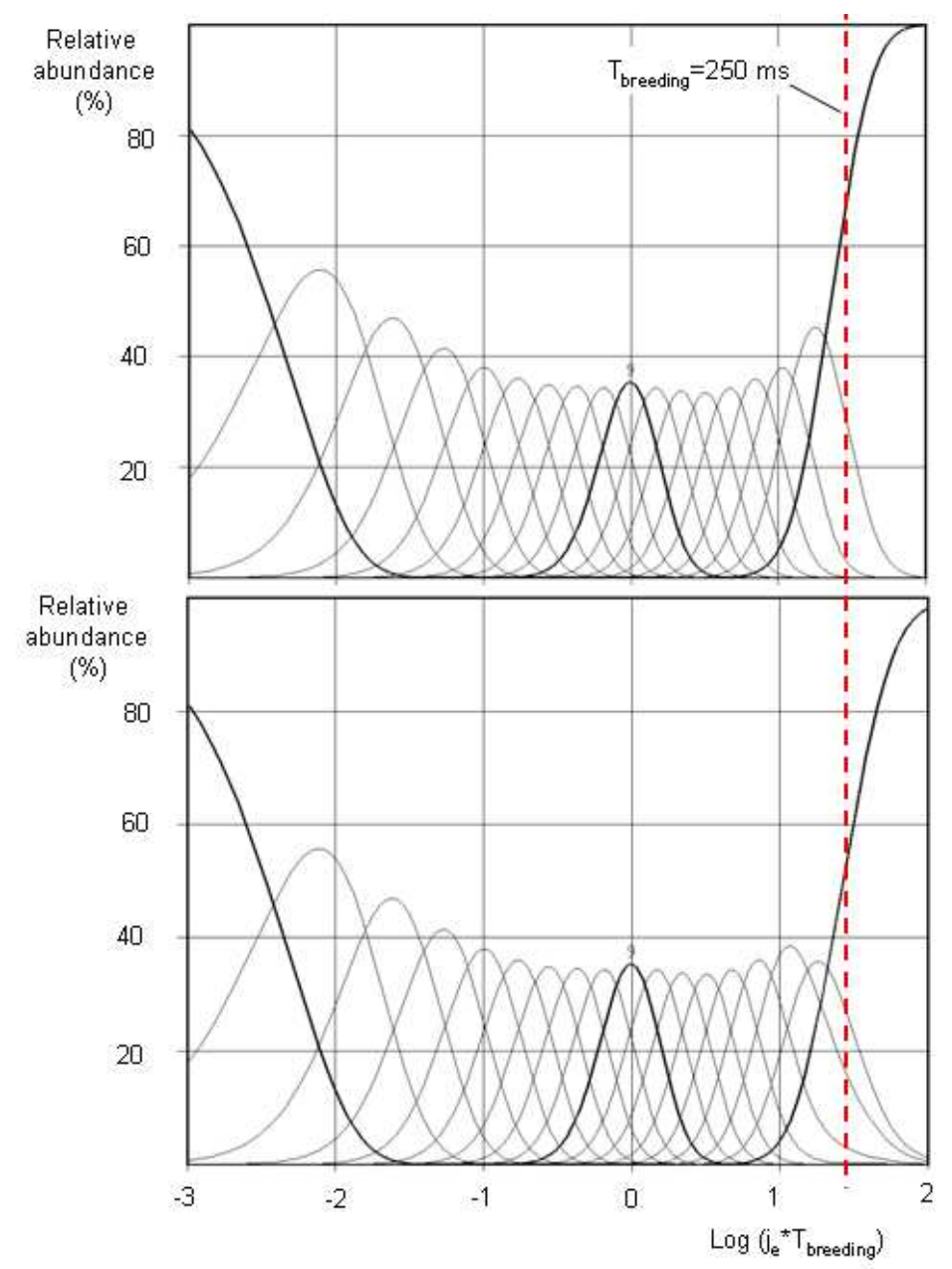

Figure 7. Potassium charge state distributions calculated with CBSIM for conditions described in the text. In the upper panel radiative recombination and charge exchange are excluded, in the lower included.

As a comparison, the transmission was measured for a normal trapping time of $20 \mathrm{~ms}$ followed by $9 \mathrm{~ms}$ breeding, resulting in a peak charge state of 10+. As can be seen in the graph the efficiency for this mode of operation was $11 \%$. The long breeding time required for closed shell breeding, resulting in a large space charge inside the trap and a very high extracted instantaneous ion beam current, precludes the use of the method at REX-ISOLDE.

A variant of the genuine closed shell breeding is nevertheless used in the daily operation of REX. Even if the machine constraints prevent an adjustment of the electron beam energy to the shell gap potential, we can still gain in transmission efficiency by breeding the ions to a closed shell configuration. For instance Ne-like copper $\left({ }^{65} \mathrm{Cu}^{19+}\right)$ could be bunched and charge bred with a total record efficiency of $19 \%$ (see figure 4). The ionization potential was significantly lower $(\sim 1500 \mathrm{eV}$ for $19+\rightarrow 20+)$ than the electron beam energy $(\sim 4300 \mathrm{eV})$. With an assumed REXTRAP efficiency of 55-60\%, the EBIS efficiency for charge state 19+ was 32-35\%. Additional information about closed shell breeding tests at REX-ISOLDE is found in [45]. 


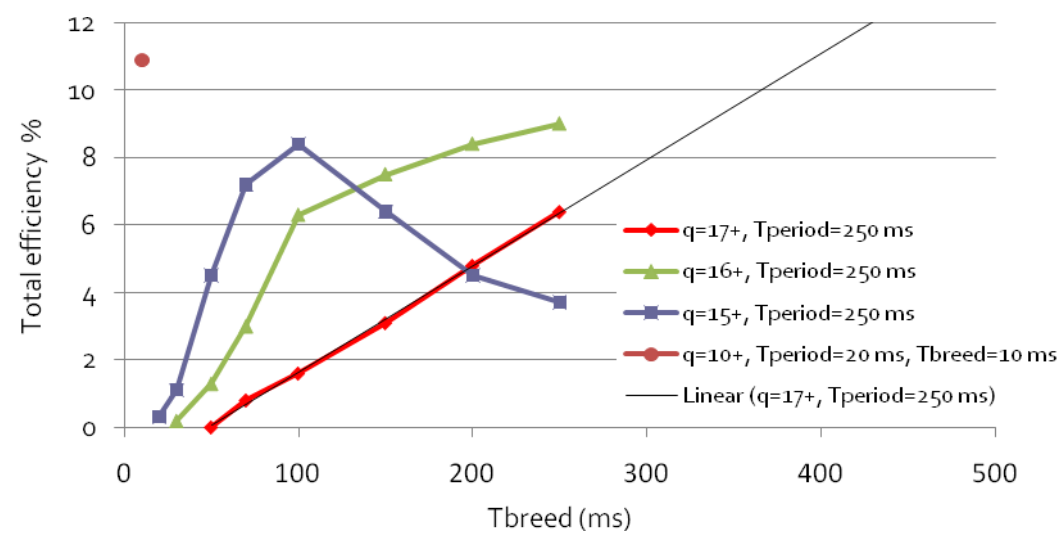

Figure 8. The combined transmission efficiency of REXTRAP and REXEBIS for different charge states of ${ }^{39} \mathrm{~K}$ as a function of breeding time when operated in closed shell mode.

\subsection{Beam contamination and cleaning}

Apart from striving for the highest possible intensity, beam purity is of crucial importance for many experiments. The level of tolerated contamination varies with the experimental setup and can be as strict as $<10 \%$ of the requested beam, particularly if particle-tagging is not available. Many experiments make use of ionization chambers and Bragg detectors for on-line beam identification. The beam contamination originates either from the primary target as an isobaric mass-resolution of the cocktail beam is rarely within reach of the separators, or from the EBIS in the form of residual gas ions, such as $\mathrm{C}, \mathrm{N}, \mathrm{O}$ and effusing Ne buffer-gas from REXTRAP. In addition Ar, which is used as venting gas, is also detected as a contaminant.

The EBIS contaminations are almost exclusively non-isobaric contaminations, but some ion species may have A/q-values not resolvable from the radioactive beam by the REX mass separator, which has an A/q-resolution of around 150. Trace elements as $\mathrm{La}, \mathrm{B}, \mathrm{Cu}$ and Ti from the cathode, collector and drift tubes may also be detected after the EBIS at unsuitable A/q-values, then at levels of some 10 to 1000 pps. The partial pressures for $\mathrm{C}, \mathrm{O}, \mathrm{Ne}$ and $\mathrm{Ar}$ in the REXEBIS trapping region when the vacuum is optimal are estimated from the extracted current to be around $3 \cdot 10^{-12}, 2 \cdot 10^{-12}$, $5 \cdot 10^{-12}$ and $4 \cdot 10^{-13}$ mbar, respectively. This corresponds to between 5 and 100 particle-pA in the A/q-peaks, while $<0.1 \mathrm{epA}$ (limit given by the Faraday cup readout) is recorded in between the peaks. A mass spectrum for beam extracted from REXEBIS is presented in figure 9 after new NEG strips were installed. The result from internal coating of the drift tubes and collector with a NEG layer was inconclusive. Even superior vacuum could be achieved with a cryogenic system, although the risk of memory effects between consecutive runs is higher as reported in [46]. The REXEBIS system needs to be baked-out after venting to atmosphere so a minimum time of a week is required for an intervention, similar to an opening cycle of a cryogenic system.

For most elements an A/q-ratio within the acceptance of the accelerator can be found without background, except for certain isotopes of low- $\mathrm{Z}$ elements such as $\mathrm{Li}, \mathrm{Be}, \mathrm{B}$, etc. In those cases single or double-stage stripping in the LINAC with carbon foils of thickness $4-50 \mu \mathrm{g} / \mathrm{cm}^{2}$ is used to separate the overlapping charge state distributions, see further [38]. Enriched ${ }^{20} \mathrm{Ne}$ as buffer gas in REXTRAP is regularly used to avoid the ${ }^{22} \mathrm{Ne}$ isotope from being superimposed on ${ }^{11} \mathrm{Be}$ or ${ }^{11} \mathrm{Li}$. 


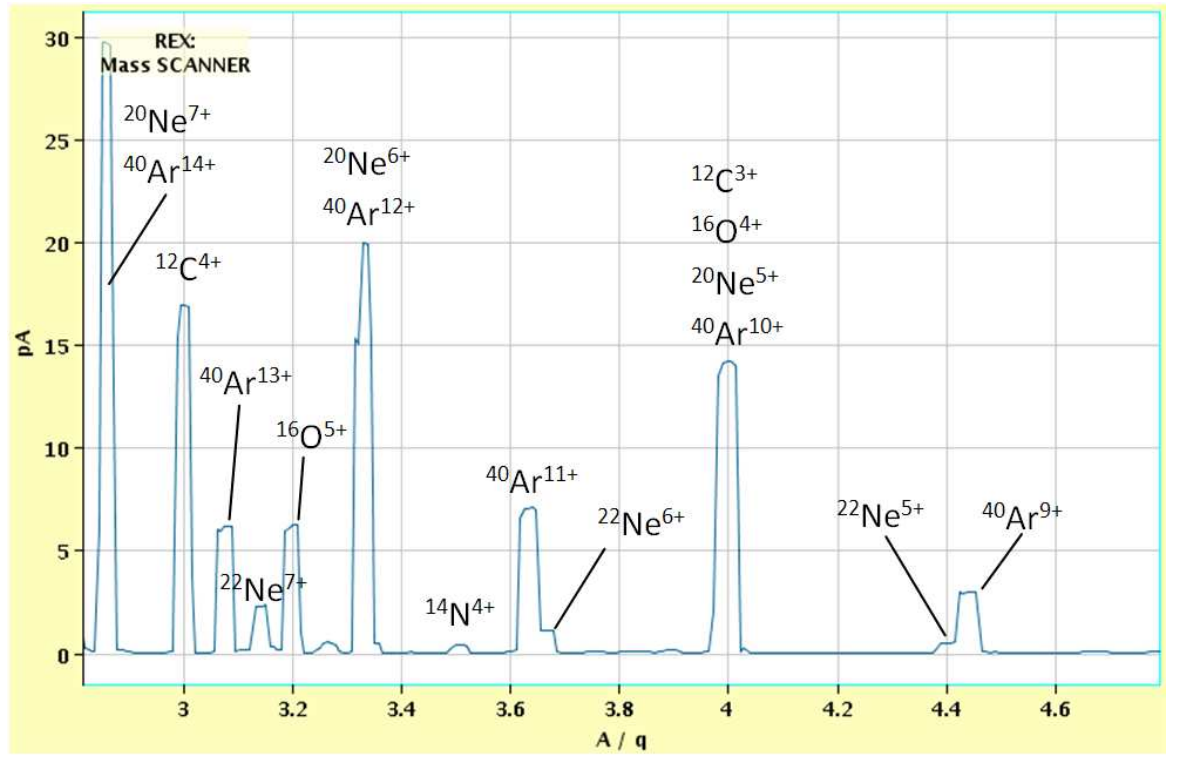

Figure 9. Extracted residual mass-spectrum from REXEBIS for optimal vacuum conditions. The Ne peaks are due to buffer-gas effusing from REXTRAP into the breeder. At the time of recording an electron beam current of $150 \mathrm{~mA}$ was used with a breeding time of $28 \mathrm{~ms}$.

Low intensity beams with less than a few 1000 pps from ISOLDE have been accelerated in REX for physics. To further improve the beam purity molecular sideband beams from ISOLDE can be used, or REXTRAP can be operated in mass resolving mode.

\subsubsection{Molecular beams}

Molecular sideband ions have been part of the ISOL-tools for a long time. The method makes use of the fact that elements along an isobar have different chemical properties and often do not form similar molecular compounds. Thereby the element can be transferred into a clean molecular sideband with a high chemical selectivity. The method is thoroughly treated in [47].

For example, instead of selecting ${ }^{96} \mathrm{Sr}$ directly from the target and primary ion source, with a substantial ${ }^{96} \mathrm{Rb}$ contamination superimposed, the molecule ${ }^{96} \mathrm{Sr}^{19} \mathrm{~F}$ was mass selected (produced by injection of $\mathrm{CF}_{4}$ gas). Rubidium does not preferably bind as $\mathrm{RbF}$, and was therefore suppressed. The SrF molecule was transferred to REX where it can either be dissociated inside the trap or the EBIS. In this particular case the primary $A=115$ beam from ISOLDE happened to contain ${ }^{115} \mathrm{In}$ and ${ }^{115} \mathrm{Ag}$, which then followed into the breeder. Nevertheless, by charge breeding strontium to $23+$ an A/q-value different from any possible mass-to-charge ratio of $\mathrm{A}=115$ was obtained. The complete procedure is illustrated in figure 10. This two-stage separation demonstrates the suppression strength of the method. The final selectivity is given by the probability of the contaminant to bind in the same molecular configuration and should preferably be tested in advance of the experiment. The REX low-energy efficiency depends on the electro-negativity of the desired ion in the molecule, disfavoring radioactive ions with high electro-negative values, since they tend to become neutral when the molecule is broken up and escape the confining potential of the electron beam. For instance, a REX low-energy record efficiency of $16 \%$ was obtained for $\mathrm{Al}^{7+}$ injected as $\mathrm{AlF}^{+}$ 


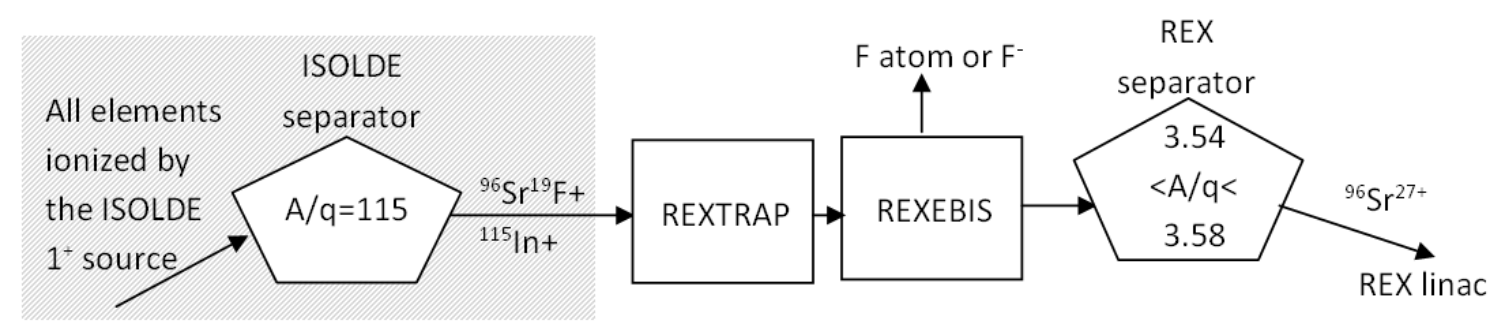

Figure 10. By extraction of ${ }^{96} \mathrm{Sr}^{19} \mathrm{~F}$ from ISOLDE the contaminating ${ }^{96} \mathrm{Rb}$ is avoided. The SrF molecule is dissociated in the EBIS and the strontium is charge bred. The contaminating ${ }^{115} \mathrm{In}$ is separated away in the REX separator.

ion into the EBIS, while only a couple of percent was achieved for $\mathrm{F}^{5+}$. A higher electron beam current density would improve the trapping probability and therefore the efficiency.

Molecular beams from the ISOLDE targets are not exclusively used to obtain pure beams, but as well to improve the release of elements that in elementary form are highly reactive with the target container or ion source surfaces, or have high vaporization temperatures. Examples are carbon, nitrogen, oxygen, fluorine and chlorine (for further reading see [48]). At REX-ISOLDE this technique was fruitfully used for extraction and breeding of ${ }^{10} \mathrm{C}$, in ${ }^{10} \mathrm{C}^{16} \mathrm{O}$ form. The molecule was broken up inside the EBIS and charge bred to $3+$ before being accelerated. Worth mentioning is the use of a stripper foil after the acceleration to separate away ${ }^{20} \mathrm{Ne}^{6+}$ from the ${ }^{10} \mathrm{C}^{3+}$ beam. Since 2005 several molecular beams have been injected and their fragments post-accelerated at REX-ISOLDE, such as ${ }^{10} \mathrm{C}$ from $\mathrm{CO},{ }^{70} \mathrm{Se}$ from $\mathrm{SeCO},{ }^{96} \mathrm{Sr}$ from $\mathrm{SrF}$ and ${ }^{140,142} \mathrm{Ba}$ from BaF. Additional information on the application of molecular sideband beams at REX is found in [49, 50].

\subsubsection{REXTRAP in mass resolving mode}

To suppress low-intensity isobaric contaminants from ISOLDE the intrinsic mass resolution of a Penning trap may be employed. By ion-motion manipulation inside the trap, see figure 11, a mass resolving power in the order of $10^{4}$ to $10^{5}$ is in principle achievable. The complete trapped ion cloud, including contaminants, is first compressed by a large amplitude quadrupolar excitation inside the Penning trap for approximately $50 \mathrm{~ms}$ and thereafter displaced radially with a dipolar mass-independent excitation during $20 \mathrm{~ms}$. Once offset, a low-amplitude quadrupolar excitation is applied for 100-200 ms, which selectively re-centres the desired ions. These can then be extracted from the trap and injected into the EBIS in a normal way, while the still displaced contaminants are stopped by a diaphragm. The complete cycle takes up to $400 \mathrm{~ms}$ and the required pulsed injection into the trap is obtained by collection and pre-bunching in the RFQ cooler.

The technique has been extensively investigated at REX, mainly with stable beams [51, 52]. A mass resolution of $3 \cdot 10^{4}$ for ${ }^{39} \mathrm{~K}$ has been demonstrated with a suppression factor exceeding 20 for nearby contaminating masses. In standard operation the mass resolution is approximately 300 and little suppression is attained. It is important to note that the modified cooling procedure inside the trap is less efficient but still adequate for successive ion injection into the EBIS, reflected in a total transmission of the REX low-energy part of up to 5\%. Increased mass resolution and suppression factor lead to lower transmission efficiencies. Different masses, varying injected currents and cooling schemes have been investigated. It has been found that the operation mode is fairly complicated 


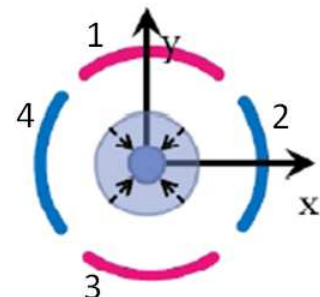

$1,3 \quad A_{1} \cos \omega_{c} t$

$2,4-\mathrm{A}_{1} \cos \omega_{\mathrm{c}} \mathrm{t}$

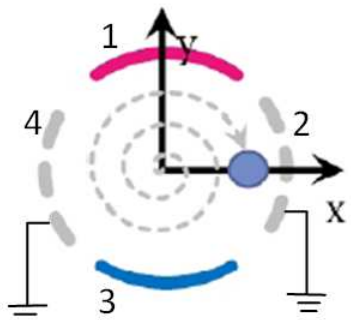

$1 A_{2} \cos \omega_{-} t$

$3-\mathrm{A}_{2} \cos \omega . \mathrm{t}$

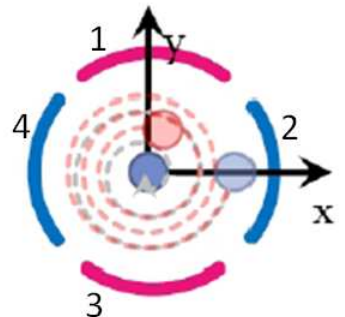

$1,3 \quad \mathrm{~A}_{3} \cos \omega_{\mathrm{c}} \mathrm{t}$

$2,4-\mathrm{A}_{3} \cos \omega_{\mathrm{c}} \mathrm{t}$

Figure 11. Sequence of RF excitations to achieve isobaric separation in REXTRAP. Left: High-amplitude quadrupolar excitation to compress the ion cloud. Middle: Dipolar excitation at magnetron frequency to displace the ion cloud outside the acceptance of the extraction diaphragm. Right: Low-amplitude massselective quadrupolar excitation to re-centre the desired ions. Amplitudes A1 $>>$ A2 $>$ A3. Picture adjusted from [51].

to set up. The mass resolution is affected by space-charge effects and above a few $10^{7}$ ions/bunch the resolution is lower than for the High Resolution Separator, i.e. 5000. With a repetition rate of $3 \mathrm{~Hz}$ this translates to a few pA injected beam, including the contaminants. The long cycle time disqualifies ions with $\mathrm{t}_{1 / 2}<100 \mathrm{~ms}$. As a conclusion, the method is not universal but a complement to molecular sideband beams and suited for cases where the highly selective Resonant Ionization Laser Ion Source (RILIS) [53] and target-ion source selectivity are not readily available.

\subsection{Continuous ion injection}

REXTRAP normally delivers a beam pulse of 3-15 $\mu$ s (FWHM), depending on trap extraction conditions and ion mass, which is transferred to REXEBIS. Although the EBIS has been designed for pulsed injection with a time-variable outer barrier, it is of general interest to investigate efficiencies and breeding times for continuous beam injection (also known as accumulation mode). In principle this mode opens up new possibilities for short-lived ions and, high intensity beams for which the Penning trap becomes a limiting factor. To be efficient, however, a high-quality emittance from the on-line separator is required as continuous injection mode has a reduced phase-space acceptance compared to pulsed injection, due to the injected beam passing over the EBIS outer barrier (for a detailed discussion see [32]). In addition, a rapid ionization from $1+$ to $2+$ or higher charge state during one round trip inside the EBIS trapping region is essential.

The continuous injection mode has been examined at REX. CW beams from either a reference ion source or ISOLDE were used. The latter had emittances in the order of $15-30 \mathrm{~mm} \cdot \mathrm{mrad}(2 \sigma$, $30 \mathrm{keV}$ ), optionally cooled by the RFQ cooler ISCOOL [54] to emittances of about 3-10 mm. mrad. The beams were shot through a non-active REXTRAP (magnetic field still on though) with $75 \%$ efficiency and injected over a slightly lowered outer barrier into the EBIS. After finishing collection and breeding, the outer barrier was fully lowered and the rapidly extracted ions mass-analyzed. An efficiency of $4 \%$ for ${ }^{39} \mathrm{~K}^{9+}$ was reached for $9.5 \mathrm{~ms}$ breeding time and $10 \mathrm{~ms}$ period time. This efficiency should be compared to a pulsed injection efficiency which can attain values of $15 \%$ (trap + EBIS) for 10+. It should be noted, however, that the CW injection showed no decrease in 


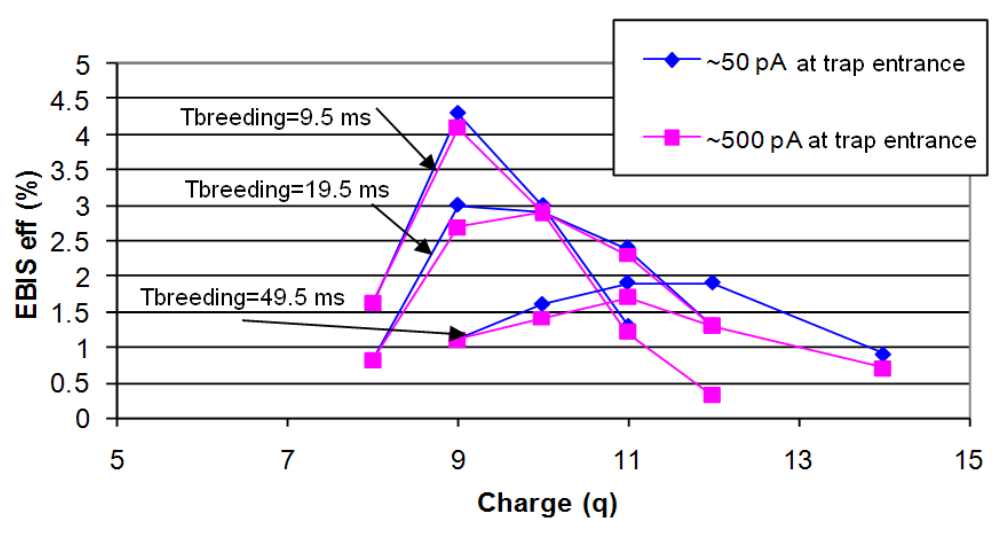

Figure 12. REXEBIS breeding efficiency as a function of the charge state for continuous injection of ${ }^{39} \mathrm{~K}$ with different beam currents and breeding times.

efficiency when the beam current was increased from 50 to $500 \mathrm{pA}$ (see figure 12), which is not the case for pulsed injection. This demonstrates the method's potential for high-intensity beams. As expected, a lower peak charge-state was recorded for the accumulation mode (9+) compared to pulsed injection (10+). A slightly higher efficiency of $5 \%$ for ${ }^{87} \mathrm{Rb}^{17+}$, breeding time $29 \mathrm{~ms}$, was achieved with a cold injected beam from ISCOOL. The higher efficiency could either be attributed to a smaller beam emittance or to a more effective trapping (ionization to $2+$ during the roundtrip) inside the EBIS. A drawback of the technique is the lack of 'beam memory reset' inside the Penning trap, meaning that the EBIS injection is sensitive to the settings of the primary target ion source (ISCOOL in case that is used) and the whole consecutive beam line. The results of these tests are more extensively discussed in $[35,45]$.

\subsection{In-trap decay}

In-trap decay is an indirect beam production technique, which was explored at REX-ISOLDE. Due to chemical reasons, refractory elements such as Fe have a long release time from the thick primary target and short-lived Fe isotopes can therefore not be delivered to experiments at ISOLDE. One way to circumvent this problem is to produce the mother isotope of the desired isotope and then let it decay in either REXTRAP or REXEBIS in order to extract the daughter ions to the experiment after the decay. This method has previously been used at ISOLTRAP with great success [55]. Pure radioactive manganese nuclei, ${ }^{61} \mathrm{Mn} \mathrm{t}_{1 / 2}=670 \mathrm{~ms}$ and later also ${ }^{62} \mathrm{Mn} \mathrm{t}_{1 / 2}=880 \mathrm{~ms}$, copiously produced by RILIS, were injected into REX in an explorative experiment [56]. The ions were trapped inside REXTRAP for various times ranging from $300 \mathrm{~ms}$ to $1100 \mathrm{~ms}$, before being transferred to REXEBIS, charge bred for $28 \mathrm{~ms}$ to $15+$, and thereafter accelerated to Miniball. In this measurement series no Fe daughter ions were detected at the experimental setup after the LINAC. On the other hand, when the breeding time was increased to $298 \mathrm{~ms}$ and 21+ ions were accelerated, a significant part of the beam consisted of Fe. This proves that recoiling daughter ions can be trapped in REXEBIS, although the experiment could not explain why the daughter trapping in the Penning trap did not work out. At REX it would be preferable if the decay can take place inside REXTRAP so the in-trap decay becomes decoupled from the breeding process and, thereby A/q-rescaling of 
Table 3. Possible isotope candidates for the in-trap decay production method.

\begin{tabular}{|c|c|c|}
\hline Daughter & Mother & $\mathbf{T}_{1 / 2}$ mother \\
\hline${ }^{12} \mathrm{~B}$ & ${ }^{12} \mathrm{Be}$ & $23.6 \mathrm{~ms}$ \\
\hline${ }^{33,34,35} \mathrm{Si}$ & ${ }^{33,34,35} \mathrm{Al}$ & $54,60,150 \mathrm{~ms}$ \\
\hline $\mathrm{Ti}$ & $\mathrm{Sc}$ & \\
\hline${ }^{61,62,63} \mathrm{Fe}$ & ${ }^{61,62,63} \mathrm{Mn}$ & $710,880,250 \mathrm{~ms}$ \\
\hline${ }^{98-103} \mathrm{Zr}$ & ${ }^{98-103} \mathrm{Y}$ & $0.23 \mathrm{~s}$ to $3.75 \mathrm{~s}$ \\
\hline
\end{tabular}

the LINAC can be avoided when the trapping time is optimized. Future tests dedicated to the understanding of possible loss-mechanisms inside REXTRAP are foreseen.

The method has restrictions of which certain can be addressed by next-generation EBIB. Firstly, recoil energies of several $100 \mathrm{eV}$ are typical; for ${ }^{61} \mathrm{Fe}$ the recoil energy distribution reached $450 \mathrm{eV}$. The recoil ions from high Q-value decays are more easily trapped in EBIB with higher magnetic field strength and larger electron beam current. Secondly, $\beta^{+}$decay is less efficient than $\beta^{-}$as a large part of the daughter ions end up as neutral atoms, although a certain part will have $1+$ or $\mathrm{q}+$ charge caused by Auger and shake-off effects. In-trap decay is limited to mother ions with limited half-life, i.e. maximum around a second. Future possible candidates, which are not yet present in the ISOLDE yield database [41], are listed in table 3. The technique is described in detail in [57] and summarized in [50].

Obviously, there are also cases when the effectiveness of the in-trap decay is counterproductive. For example, an experiment requesting post accelerated ${ }^{9} \mathrm{Li}\left(\mathrm{t}_{1 / 2}=178 \mathrm{~ms}\right)$ detected a fraction of the decay product ${ }^{9} \mathrm{Be}$ in the beam as well. Likewise, for an ${ }^{80} \mathrm{Zn}$ beam, a contamination of ${ }^{80} \mathrm{Ga}$ was seen.

\section{Other EBIB systems dedicated to post-acceleration or trap injection}

After being the only operational EBIS/T based charge breeder for radioactive ions for several years REXEBIS has now been joined by a number of devices, some operational while others still being in the design or commissioning phase. These breeders are briefly introduced below.

\subsection{EBIB for post-acceleration}

The RHIC TestEBIS $[58,59]$ has demonstrated a significant increase of the electron beam current compared with other EBIS devices. The current has been raised one order of magnitude and 6-8 A electron beams for durations of $100 \mathrm{~ms}$ from a semi-immersed gun are common. For shorter pulsing times beams of 10-13 A at typical electron energies of $20 \mathrm{keV}$ have been propagated. The RHICEBIS itself will not handle radioactive ions but feed a heavy-ion LINAC followed by synchrotrons with different stable elements, the most challenging being $\mathrm{Au}^{32+}$. As the rapidity of the breeding is not of major importance, a moderate electron beam current density of $575 \mathrm{~A} / \mathrm{cm}^{2}$ has been chosen, producing the desired gold ions in $35 \mathrm{~ms}$. The EBIS is capable of producing at least 3.4.109 $\mathrm{Au}^{32+}$ per pulse. Although the EBIS has not been specifically designed as charge breeder 
system, it is essentially using the principle. The reason behind is the requested pulse-to-pulse switching of the extracted beam and therefore the EBIS will make use of external ion injection. For instance the 1+ gold ions are injected from a liquid metal ion-source. Until now no dedicated injection efficiency studies have been performed, but the otherwise impressive performances of this EBIS show the possibilities of high-capacity radioactive breeders.

The National Superconducting Cyclotron Laboratory (NSCL) at Michigan State University (MSU) is currently constructing ReA3 [60], a re-accelerator for rare isotopes produced by projectile fragmentation and in-flight fission which are thereafter thermalized in a gas stopper. NSCL is the only place in the world currently implementing a re-accelerator at an in-flight rare isotope facility. The main components are a hybrid EBIS/T as charge breeder, a mass-over-charge separator, a room-temperature RFQ cavity and a superconducting drift-tube LINAC. In contrast to REX-ISOLDE a bunching Penning trap is not situated in front of the breeder but continuous injection of $60 \mathrm{keV}$ singly charged ions decelerated to a few hundred $\mathrm{eV}$ is employed. The MSU EBIT $[61,62]$ has been designed in collaboration with MPIK in Heidelberg and ISAC/TRIUMF and is based on the TITAN EBIT (see below), although changes have been implemented in order to adapt it to breeding for re-acceleration. The main changes compared to the TITAN EBIT are an improved electron gun design and a different magnetic field configuration. Two high-compression electron guns have been designed, with anticipated electron beams of 1.5 and $2.5 \mathrm{~A}$.

Apart from a high electron current, a large-diameter electron beam, not necessarily with highest electron density, is preferable for optimum acceptance of the injected continuous ion beam in the EBIT. However, for fastest charge state breeding a high electron density, and thus a small beam diameter, is required. To fulfill the contradicting requirements extensive injection simulations have been performed $[63,64]$ and a hybrid magnet featuring a short $6 \mathrm{~T}$ field region for fast and final charge breeding and an extended field region with variable field strength to optimize the acceptance of the system has been chosen, see figure 13 .

As the bore is at cryogenic temperature the EBIT will operate in ultra-high vacuum resulting in very low levels of contamination in the ion beam. The platform potential is changed prior to the pulsed extraction to deliver the ions with the correct injection energy to the LINAC. As an upgrade option two EBITs in push-pull mode could deliver a pseudo-continuous beam; while one is accumulating and breeding the other is slowly extracting ions. Even using closed shell breeding in order to reach efficiencies above $50 \%$, breeding times of less than $20 \mathrm{~ms}$ are expected as an electron density approaching $10^{4} \mathrm{~A} / \mathrm{cm}^{2}$ should be attainable. With an electron beam current of about $1.5 \mathrm{~A}$ available the NSCL EBIT will be able to hold $10^{10}$ positive charges inside the trap while only partially compensating the charge from the electron beam. For repetition rates of 10$100 \mathrm{~Hz}$, beam rate capabilities of the order of $10^{10}$ ions/s and possibly above are expected. The EBIT is presently in the commissioning phase.

The CAlifornium Rare Ion Breeder Upgrade [65] is a radioactive beam facility for the Argonne Tandem Linac Accelerator System (ATLAS). Fission fragments from an up to $1 \mathrm{Ci}$ strong ${ }^{252} \mathrm{Cf}$ source are thermalized and collected by a helium gas catcher. The cooled beam is mass-separated and presently fed to a $10.5 \mathrm{GHz}$ ECRIS for charge breeding before being post-accelerated by ATLAS. The setup is in its final phase of commissioning and already a record ECRIS charge breeding efficiency of $>11 \%$ for ${ }^{85} \mathrm{Rb}^{17+}$ has been demonstrated. Nonetheless, to further increase the delivered experimental beam and improve the beam purity a conversion to an EBIS is considered as 


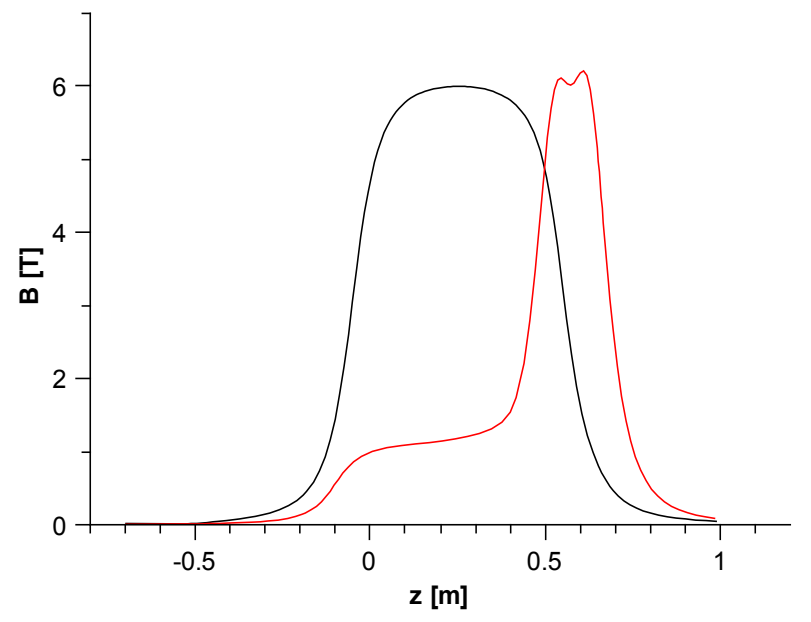

Figure 13. Magnetic field profiles for MSU EBIT created by a combination of an extended solenoid and a Helmholtz coil configuration. The field can be varied to optimize beam injection and breeding time.

a higher $\mathrm{Cf}$ source intensity has many difficulties associated to it. Argonne has initiated a collaboration with Brookhaven National Laboratory to test breeding efficiencies of low-intensity beams and study shell closure effects using a low-current (0.2 or $2 \mathrm{~A}$ ), high-perveance electron gun [66]. The EBIS would be installed after the mass separator system with an RFQ cooler in between and replace the present ECRIS breeder.

\subsection{EBIB for high-precision mass measurements}

A few setups exist that use EBIT as charge breeder to feed mass measurement systems, namely TRIUMF's Ion Trap for Atomic and Nuclear science (TITAN) and GSI's Highly-charged Ion Trap (HITRAP), and soon also precise Measurements on very short-lived nuclei using an Advanced Trapping System for highly-charged ions (MATS) to be installed at the future FAIR facility. The nuclear binding energy can be precisely derived from mass measurements with a precision of $10^{-8}$ or higher. As previously mentioned, the accuracy of mass determinations in Penning traps is proportional to the charge and although only a few ions are required per measurement cycle, the efficiency should nonetheless be high, as many of the ions are exotic and produced in small numbers.

The TITAN EBIT was the first EBIT-based charge breeder [67]. The system has been designed and built in collaboration between TRIUMF and MPIK at Heidelberg. The system will charge breed on-line isotopes, with half-lives considerably shorter than $100 \mathrm{~ms}$ and quantities down to around 100 ions per second, provided by the Isotope Separator and ACcelerator (ISAC) and send them to the TITAN ion-trap facility [68]. In the traditional way the mass is determined from time-of-flight ion cyclotron resonance of the ion in the homogenous magnetic field of the Penning trap. The TITAN EBIT is based around two Helmholtz coils with a maximum magnetic field of 6 Tesla and has presently a design electron current of $0.5 \mathrm{~A}$, corresponding to a current density of $30 \mathrm{kA} / \mathrm{cm}^{2}$ in the trapping region. A few features make this breeder special, such as the gun being retractable and replaceable by silicon detectors (for $\alpha$ - and $\beta$-decay detection) and multi-channel plate detectors. That is to say, the EBIT can be used for electron capture studies when no electron beam is present and the ions are trapped as in a Penning trap. In addition, the central trapping region kept at cryo- 


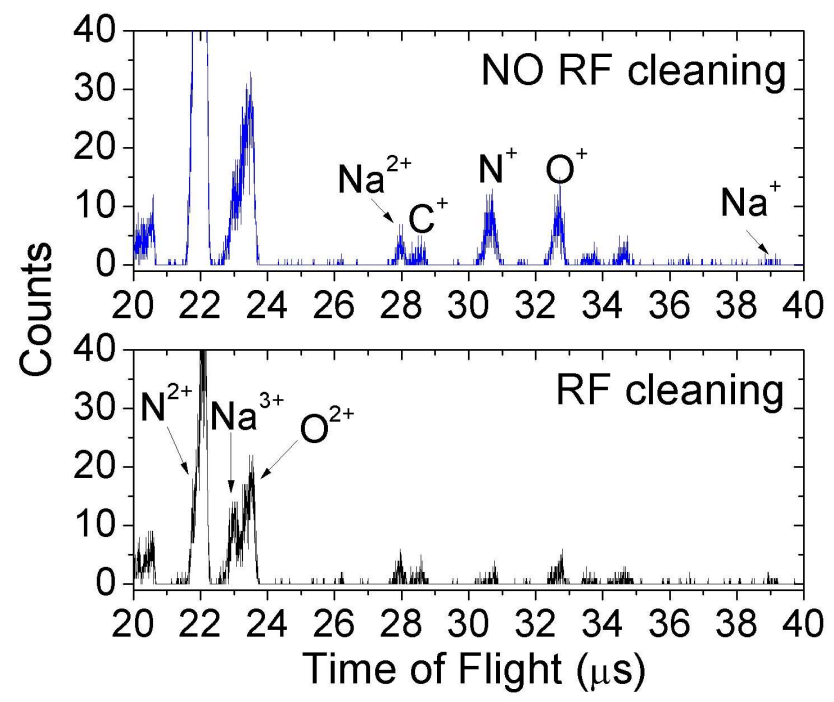

Figure 14. First example of trap cleaning from the TITAN EBIT where $\mathrm{N}^{+}$was expelled.

genic temperature has an eight-fold, azimuthally segmented electrode, facilitating measurement of induced currents on the electrodes for ion identification and, RF excitation for ion expulsion. The trap cleaning method had already successfully been demonstrated at the BRIC breeder [69] and was thereafter tested with the TITAN EBIT when a $\mathrm{N}^{1+}$ contamination was removed from the EBIT using millisecond RF excitation (see figure 14). This method is of interest but remains to be tested also for higher electron currents when the radial space-charge will seriously affect the excitation. In April 2009, the first radioactive ions $\left({ }^{25} \mathrm{Na}, \mathrm{t}_{1 / 2}=59.1 \mathrm{~s}\right)$ were charge bred. The ions were bunched in the preceding RFQ cooler and with a modest electron beam of $5 \mathrm{~mA}$ charge states $2+$ and $3+$ were obtained after $60 \mathrm{~ms}$ breeding time. Argon that had been injected into the system as gas a couple of weeks before was still visible in the extraction spectrum. The memory effect for ion-injected beams is expected to be smaller because of less interaction with the cold walls. First on-line results from TITAN EBIT have been published in $[46,70]$.

Although not dedicated to radioactive beams, the HITRAP facility [71] has demonstrated that charge breeding of stable beams can be carried out also with a compact commercial EBIT source. The SPARC EBIT in use is a small SmCo permanent-magnet device from DREEBIT [72], which can provide electron currents up to $50 \mathrm{~mA}$. The off-line device is aimed to feed HITRAP with highly charged ions. The source has been designed for gas injection through a needle valve and to add to the number of beams external ion injection has been tried. For the breeding test singly charged potassium ions were injected during $20 \mathrm{~ms}$. After a $3 \mathrm{~s}$ breeding time the distribution had reached its equilibrium. A charge state distribution peaking at 17+ was recorded after the source, but even some fully stripped 19+ potassium ions could be detected. The total capture efficiency was estimated to $\sim 2 \cdot 10^{-4}$ and the charge breeding efficiencies for the individual charge states are listed in table 4 for varying breeding times [73, 74]. Even if the efficiencies are modest, the test shows that also small devices can be used as stable beam breeders.

In the same way the upcoming project MATS [75] is geared towards physics with trapped radioactive ions and not post-acceleration. The experiment is to be installed at the low-energy 
Table 4. SPARC EBIT charge breeding results for potassium for various breeding times. The listed $q$ is the optimum charge for the given breeding time and $\mathrm{N}\left(\mathrm{K}^{q+}\right)$ and $\mathrm{N}(\mathrm{K})$ denote the number of extracted potassium ions in $\mathrm{q}+$ charge state and the total number of extracted ions, respectively.

\begin{tabular}{|c|c|c|c|}
\hline $\mathrm{q}$ & $\mathrm{T}_{\text {breeding }}(\mathrm{ms})$ & $\begin{array}{c}\text { Charge state distribution } \\
\mathrm{N}\left(\mathrm{K}^{q+}\right) / \mathrm{N}(\mathrm{K})(\%)\end{array}$ & $\begin{array}{c}\text { Efficiency }\left(10^{-5}\right) \\
1+\rightarrow \mathrm{q}+\end{array}$ \\
\hline $9+$ & 50 & 29 & $6.3 \pm 1.6$ \\
\hline $14+$ & 300 & 32 & $7.2 \pm 1.8$ \\
\hline $17+$ & 1000 & 45 & $15.3 \pm 3.8$ \\
\hline
\end{tabular}

branch of the future Superconducting Fragment Separator at GSI and will make use of the broad spectrum of short-lived isotopes produced. In the present layout the in-flight separated isotopes will be stopped in a gas-catcher and mass separated before passing a second-generation coolerbuncher. Thereafter follows an advanced combination of an EBIT for charge breeding, ion traps for beam preparation, and a high-precision Penning trap (see figure 15). It should be noted that the cooler/buncher is operated at cryogenic temperatures in order to achieve minimal emittances and an effective injection into the EBIT. The EBIT itself will be designed for a high electron energy of up to $350 \mathrm{keV}$. Thus, fully stripped uranium can theoretically be produced and the device can be complementary to the HITRAP setup. Thanks to the open geometry and visual access of the trapping center of the EBIT, very different from for example the REXEBIS, the EBIT can serve as a stand-alone experimental tool. Equipped with a high-resolution X-ray spectrometer or accessed by laser, spectroscopic studies of the ions in the trapping region can be performed. Apart from that, with for instance a VUV spectrometer and a fluorescence detection system, the charge breeding process can be monitored in time.

\section{Future RIB projects and upgrades}

In order to establish the forthcoming requirements for EBIB, a short summary of three future RIBfacilities where EBIS/T are foreseen to be used is given below. The projects are: HIE-ISOLDE an upgrade of the CERN ISOL-facility; FRIB - a fragment separator recoil facility at MSU; and EURISOL - a future ultimate ISOL-facility in Europe.

Nearest in the future is the upgrade of the ISOLDE radioactive beam facility. The first part of the High Intensity Energy ISOLDE project, the energy upgrade, was approved by the CERN Research Board in December 2009. Already in 2008 a study of superconducting quarter-wave cavities to boost the REX beam energy was initiated. A preliminary design of a cryomodule containing 5 cavities and a superconducting solenoid is presented in figure 16. The superconducting cavities will be added to the existing room-temperature RFQ and IH-structure cavities. Apart from a higher energy (10 MeV/u for $\mathrm{A} / \mathrm{q}=4.5$, higher for lighter ions) of the post-accelerated beams, more intense radioactive beams from the primary target are expected with increased proton intensity on the target. Presently, the $1.4 \mathrm{GeV}$ driver beam is limited to an average current of $2 \mu \mathrm{A}$ by radiation protection reasons, whereas with improvements of the driver beam (that is LINAC4 [76] and PS Booster) and the target shielding and handling, a maximum of $5.3 \mu \mathrm{A}$ can be expected. This leads 


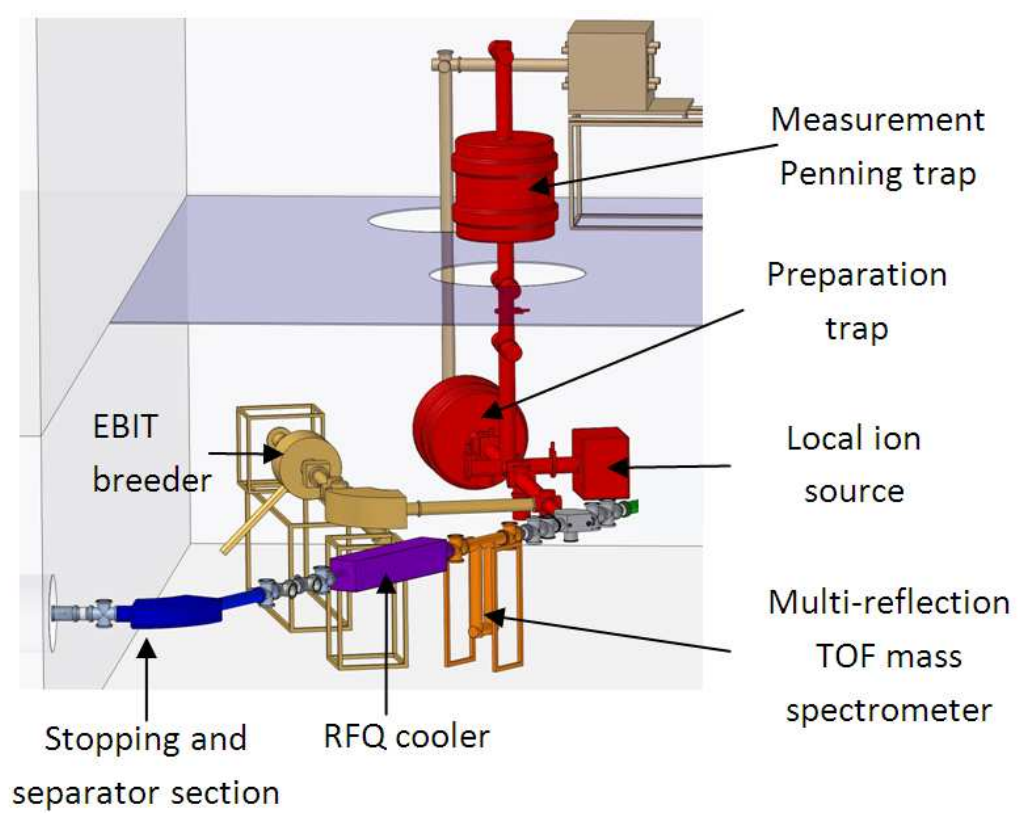

Figure 15. The layout of the MATS setup including gas catcher, RFQ cooler, EBIT breeder and Penning trap.

to exotic beam intensities from the primary target as high as $3.4 \cdot 10^{11}{ }^{127} \mathrm{Cs}$ ions/s (one of the most abundantly produced nuclei at ISOLDE), and consequently the ion throughput of the REX low-energy system will become a bottleneck. In addition, access further out towards the driplines results in isotopes with short life-times. An upgrade of REXEBIS is therefore considered and the details are discussed below.

The upcoming Facility for Rare Isotope Beams (FRIB) at MSU will be based on a $400 \mathrm{~kW} 200$ $\mathrm{MeV}$ heavy ion driver that will provide fast, stopped and re-accelerated beams [77]. The projectile fragmentation method in use gives as such access to extremely short-lived nuclei, down to the $\mu$ s region. Furthermore, the method is chemically unselective (gas stopper excluded) and can thus provide elements not available at ISOL facilities. However, a charge breeder will only be employed for the re-accelerated beams, in the same way as for the ReA3 accelerator described above, where a gas stopper precedes the breeder. Thereby the time constraint is relaxed and a few ms breeding time is acceptable. Beam intensities above $10^{8}$ ions/s are foreseen. Optionally an ISOL-target station will be included to FRIB and thereby extremely high RIB intensities are expected, in certain cases they are predicted to exceed $10^{12}$ ions/s. In order to fully and safely use these intense beams the technologies for beam manipulation after production have to be pushed to maximum performance and the design of a next-generation EBIT has therefore already started (see section below).

If one looks further into the future, the proposed EURISOL is an ultimate ISOL-facility, with its different aspects described in the EURISOL Design Study [78]. It aims to post-accelerate very rare and low-intensity radioactive nuclear beams, but also very intense beams, up to $100 \mathrm{MeV} / \mathrm{u}$ using a superconducting LINAC suited to $\mathrm{CW}$ operation. The exotic beams will have intensities of less than $10^{6}$ pps and life-times down to some ms. The intense neutron-rich beams on the other hand, for example ${ }^{132} \mathrm{Sn}$ from a multi-megawatt uranium carbide target, will be produced 


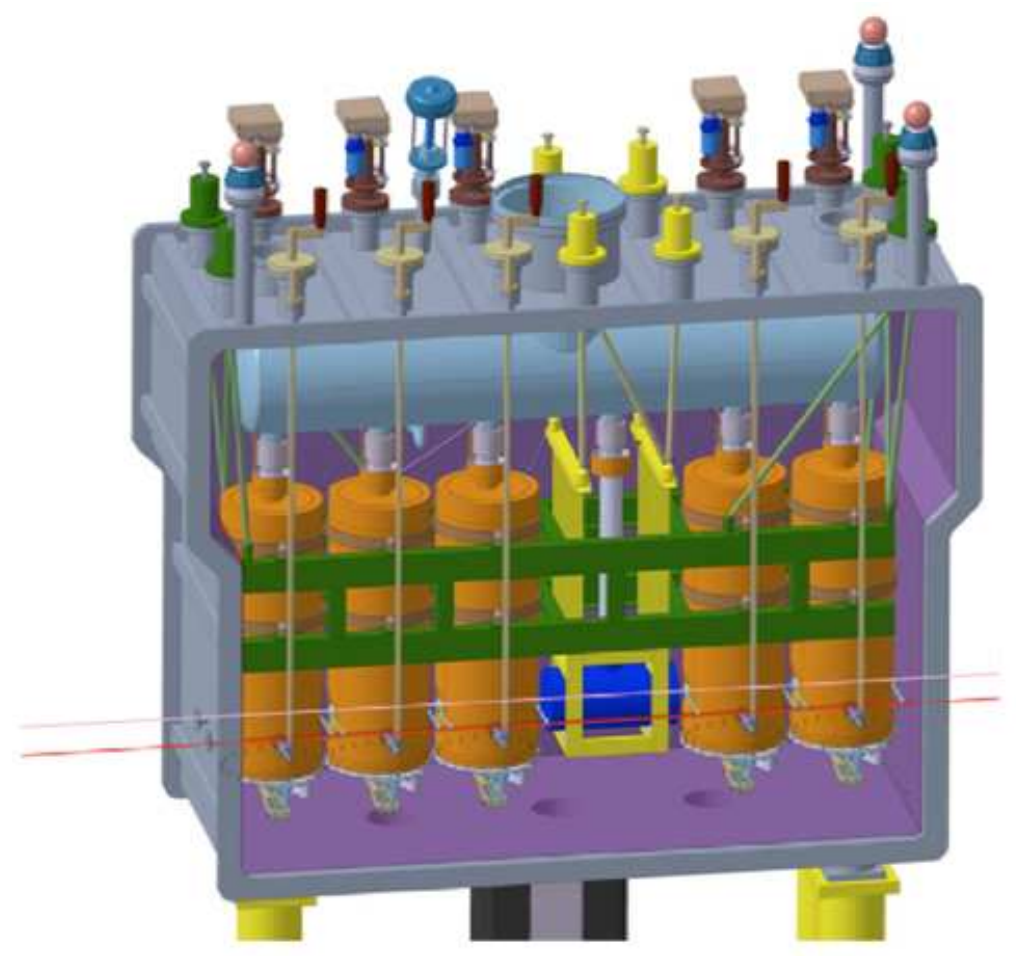

Figure 16. A cryomodule for the HIE-REX upgrade containing 5 high- $\beta$ cavities and a superconducting solenoid.

with up to $10^{13}$ ions per second and used for producing even more neutron rich nuclides by 'cold fragmentation'. The large intensity span has called for a complementary approach, using EBIB for the exotic elements in order to maintain the beam purity and ECRIS for the high intensity beams [79].

\section{Areas of improvement for EBIB}

With the wide experience gained at REX-ISOLDE, a number of operational issues have been identified that should be addressed with an upgraded breeder. The improvements should also cover the implications of the HIE-ISOLDE project. The issues, and their corresponding actions, will be briefly outlined below. Thereafter, an exceptionally performing EBIT breeder, presently in the early planning stage, will be discussed. The latter, endorsed by the Department of Energy, US, is a high-current breeder to be developed and constructed at MSU in collaboration with a number of other institutes [62].

The subjects to be improved at the REX low-energy stage are in order of priority:

Reliability. Of highest priority for REX is the need for a reliable cathode, which is able to deliver the desired current for a complete running period, that means normally 8 months. A vacuum separable gun chamber to allow for swift exchange of the gun in case of cathode failure is mandatory. 
Efficiency. An increased efficiency could be obtained with a higher electron beam current as the transverse acceptance would be larger. Furthermore, closed shell breeding could become a serious option with a larger beam current in combination with a higher current density as the required breeding time would be kept reasonable and the larger radial holding potential would reduce ion losses.

CW extraction. Ideally, one would like to have the possibility to deliver a $\mathrm{CW}$ beam, but as the REX RFQ and IH-structure will be kept, the 10\% duty factor will remain. Nevertheless, a more uniform slow extraction is best attained using more trapping drift tubes than the 3 existing in REXEBIS.

Breeding time. A shorter breeding time, and thereby higher repetition rate has been requested, partly to reduce the instant high number of ions in the extracted bunches, but also to avoid excessive half-life losses. Presently, the total hold-up time for the heaviest neutron-rich isotopes exceeds $600 \mathrm{~ms}$. With continuous ion-injection into REXEBIS and a higher current density the time should be curbed to a few $10 \mathrm{~ms}$.

Radiation aptness. REXEBIS is placed in a class $\mathrm{C}$ radiation zone. Nonetheless, it is problematic to remove contaminated parts from the zone for re-machining or maintenance and, the opening of possibly contaminated beam-lines have to be surveyed by the radioprotection group. The policy has therefore been to not accept high-intensity, long-lived $\left(t_{1 / 2}>30\right.$ days) beams for acceleration as the inherent losses may pose contamination issues at the moment of maintenance. This may change as for example several heavier $\alpha$-emitting elements, originating from high-profile physics, with long-lived daughters in the decay-chains are requested. A future breeder should be more modular and the use of replaceable elements with a minimum need for human intervention during repair and maintenance is mandatory.

CW injection. It has been shown that REXTRAP is performing well and can be used for diverse advanced ion manipulation. Nevertheless, the combined trap-EBIS system is relatively complicated and the Penning trap poses an upper intensity limit of around $10^{8}$ ions/bunch, typically translating to $10^{9}$ ions/s for a medium-Z element. For these reasons continuous ioninjection is a preferable solution. An adapted electron beam profile to increase the transverse 1+ ion acceptance should be considered. The ISOLDE RFQ cooler operated in CW mode can handle beams of several nA and should be placed in the vicinity of the EBIS in order to avoid emittance growth in the transfer line.

Space-charge capacity. In order to be able to accept all HIE-ISOLDE beam intensities an increase of the space-charge capacity is necessary. A beam current of $3 \mathrm{~A}$ and a compensation factor of 0.5 would correspond to $1.5 \cdot 10^{11}$ charges. With the increased repetition rate (limited to $100 \mathrm{~Hz}$ by the LINAC), the system is capable of handling around $4 \cdot 10^{11}$ ions. On the other hand, the priority of an increased capacity is low as most requested beams are low-intensity isotopes along the drip-lines. Thus, the high-capacity Phoenix ECRIS breeder with its higher $\mathrm{A} / \mathrm{q}$-values has not come in question as it would require a major adaption of the REX LINAC, for example a modified IH-structure. 
At present the electron beam concept to be used for an upgraded REXEBIS is not decided: either a semi-immersed high-current gun as employed in the RHIC EBIS with a current density limited to some $1000 \mathrm{~A} / \mathrm{cm}^{2}$, or an high-compressed, electrostatically focused, electron beam as in a traditional EBIT. An attractive alternative would be the aforementioned next-generation EBIT. After construction and commissioning at MSU, the EBIT is planned to be installed at ISAC for on-line performance tests with intense radioactive beams. In view of the high radioactive beam intensities readily available, ISAC/TRIUMF is an ideal test location, and with the proposed ARIEL upgrade [80], even higher intensities could be available. The breeder itself will be based on the MSU EBIT for ReA3, but with enhanced electron beam characteristics. An electron beam of $>10$ A has been proven feasible [58] and is the key to fast breeding and for providing a large ion storage capacity up to $5 \cdot 10^{11}$ charges. For $\mathrm{q}=50+$ this allows $10^{10}$ ions to be stored simultaneously. With a repetition rate of $100 \mathrm{~Hz}$ an ion throughput of $10^{12}$ ions per second would be achievable and even higher numbers for lighter ions. The initial concept is making use of a Helmholtz coil and solenoid combination with a magnetic field of $9 \mathrm{~T}$. The goal for the electron beam density is $10^{5} \mathrm{~A} / \mathrm{cm}^{2}$.

\section{Conclusions and alternatives}

Charge breeding of stable and exotic isotopes offers unique possibilities for nuclear and atomic physic experiments at both low and high energies. Questions about the prospects of the REXISOLDE project were raised in the late nineties due to the relatively complicated bunching-breeder system employed, and a preference for ECRIS based breeders governed among the laboratories. Since then a wind of change has blown and the suitability of an EBIS/T as charge breeder for radioactive ions has been proven. Existing devices already demonstrate mass-to-charge-state ratios of 2 to 4.5 for hold-up times below $1 \mathrm{~s} \mathrm{(comparable} \mathrm{to} \mathrm{typical} \mathrm{diffusion-effusion} \mathrm{times} \mathrm{of} \mathrm{an} \mathrm{ISOL}$ target - ion source). Efficiencies above 5\% for any $\mathrm{A}$ and $\mathrm{Z}$ are readily available, and intensities ranging from a few up to $10^{9}$ ions/s have been transmitted with a maintained good beam quality.

Nevertheless, the more robust and simple stripping option has several attractive advantages [8], the main being the rapidity and the lack of residual gas ions from the breeding process in the beam. Moreover, a large number of laboratories are still pursuing the development or commissioning of ECRIS charge breeders, for example ISAC/TRIUMF, SPES at Legnaro, SPIRAL2/GANIL and the CARIBU project at ANL to mention a few. LPSC, Grenoble, is developing an advanced ultrahigh vacuum and radiation compatible charge breeder, the so-called A-Phoenix [81], based on the Phoenix breeder used at ISAC and tested at ISOLDE. The main focus of the ECRIS community is to curb the contaminating background current (as high as a few nA after a simple magnetic separator), increase the maximum charge state (the A/q-value is now higher than 8 for heavier masses) and if possible lower the hold-up time (presently some $100 \mathrm{~ms}$ in CW mode). The space-charge capacity of this type is already superior to any EBIB.

As described above the second generation EBIB has significantly improved performances in terms of capacity, charge breeding time and beam diagnostics. The vigorous ongoing research and development program promise that the challenges of the upcoming RIB facilities are adequately addressed. With HIE-ISOLDE approved and several other EBIB-based facilities under way, the EBIS/T will continue to play a key-role as breeder in radioactive facilities. 


\section{Acknowledgments}

The author would like to thank colleagues that kindly have provided input to this paper, namely J. Crespo Lopez-Urrutia, F. Herfurth, O. Kester, S. Kondrashev, A. Lapierre, S. Schwarz and D. Voulot.

\section{References}

[1] I. Bergström, C. Carlberg, T. Fritioff, G. Douysset, J. Schönfelder and R. Schuch, SMILETRAP - A Penning trap facility for precision mass measurements using highly charged ions, Nucl. Instrum. Meth. A 487 (2002) 618.

[2] H. Beyer and V. Shevelko, Introduction to the Physics of Highly Charged Ions, Series in Atomic Molecular Physics, Vol. 3, Taylor \& Francis (2003).

[3] F.J. Currell ed., The physics of multiply and highly charged ions, vol. 2, Interactions with matter, Kluwer Academics Publishers (2003).

[4] P. Longevialle ed., Advances in mass spectrometry, proceedings of $11^{\text {th }}$ Int. Mass Spectr. Conf., vols. $11 \mathrm{a}$ and 11b, Bordeaux (1988).

[5] C. Carlberg et al., The SMILETRAP facility, Physica Scripta T59 (1995) 196.

[6] M. Harrison, S. Peggs and T. Roser, The RHIC accelerator, Ann. Rev. Nucl. Part. Sci. 52 (2002) 425.

[7] B. Autin et al., The acceleration and storage of radioactive ions for a neutrino factory, J. Phys. G 29 (2003) 1785 [physics/0306106].

[8] F. Wenander, Charge breeding techniques, CERN-AB-2004-035 note.

[9] E. Beebe, L. Liljeby, A. Engstrom and M. Bjorkhage, The Stockholm Electron Beam Ion Source, Physica Scripta 47 (1993) 470.

[10] B. Gastineau, J. Faure and A. Cortois, Dione, a new EBIS under construction at Saturne, Nucl. Instrum. Meth. B 9 (1985) 538.

[11] E. Beebe et al., Test EBIS operation and component development for the RHIC EBIS, J. Phys. Conf. Ser. 2 (2004) 164.

[12] Y. Blumenfeld, Radioactive beam facilities in Europe, Nucl. Instrum. Meth. B 266 (2008) 4074.

[13] H. Sakurai, Present and future radioactive ion beam facilities in the Middle East and East, Nucl. Instrum. Meth. B 266 (2008) 4080.

[14] F. Wenander, Charge breeding techniques, Nucl. Phys. A 746 (2004) 40.

[15] T. Lamy, J. Angot, T. Thuillier, European research activities on charge state breeding related to radioactive ion beam facilities, Rev. Sci. Instrum. 79 (2008) 02A909.

[16] F. Wenander, Charge state breeders: on-line results, Nucl. Instrum. Meth. B 266 (2008) 4346.

[17] O. Kester, Charge breeding application of EBIS/T devices, AIP Conf. Proc. 1099 (2009) 7.

[18] R.E. Marrs, S.R. Elliott and D.R. Knapp, Production and Trapping of Hydrogenlike and Bare Uranium Ions in an Electron Beam Ion Trap, Phys. Rev. Lett. 72 (1994) 4082.

[19] Z. Ke et al., A cooler ion trap for the TITAN on-line trapping facility at TRIUMF, Hyperfine Interactions 173 (2006) 103. 
[20] F. Wenander, EBIS as charge breeder for radioactive ion beam accelerators, Nucl. Phys. A 701 (2002) 528.

[21] H. Ravn and B. Allardyce, On-line Mass Separators, in Treatise on Heavy-Ion Science, vol. 8, D.A. Bromley ed., Plenum Press, New York (1989).

[22] M. Wada et al., Slow RI-beams from projectile fragment separators, Nucl. Instrum. Meth. B 204 (2003) 570.

[23] F. Wenander, J. Lettry and M. Lindroos, Transverse emittance investigation of the ISOLDE target ion source, Nucl. Instrum. Meth. B 204 (2003) 261.

[24] E. Beebe et al., External ion injection into an EBIS source: efficiency measurements, Nucl. Instrum. Meth. B 93 (1994) 378.

[25] B. Visentin, P. Van Duppen, P. A. Leroy, F. Harrault, R. Gobin and ISOLDE Collaboration, Efficiency measurements for a low charge state ionic injection into an electron beam ion source, Nucl. Instrum. Meth. B 101 (1995) 275.

[26] D. Habs et al., The REX-ISOLDE project, Hyperfine Interactions 129 (2000) 43.

[27] F. Ames, J. Cederkall, T. Sieber and F. Wenander, The REX-ISOLDE Facility: Design and Commissioning Report, CERN-2005-009.

[28] P. Van Duppen and K. Riisager, REX-ISOLDE: from experiment to facility, topical review to be published in J. Phys. G.

[29] J. Eberth et al., Miniball - A Ge Detector Array for Radioactive Ion Beam Facilities, Prog. Part. Nucl. Phys. 46 (2001) 389.

[30] P. Schmidt, REXTRAP - Ion Accumulation, Cooling and Bunching, Doctoral Thesis, Johannes Gutenberg-Universitat Mainz (2007).

[31] F. Ames et al., Cooling of radioactive ions with the Penning trap REXTRAP, Nucl. Instrum. Meth. A 538 (2005) 17.

[32] F. Wenander, B. Jonson, L. Liljeby and G. Nyman, REXEBIS - the electron beam ion source for the REX-ISOLDE project, CERN-OPEN-2000-320.

[33] P. Delahaye, F. Ames and A. Kellerbaeur, Study of the charge exchange process at low energy with REXTRAP, Nucl. Phys. A 746 (2004) 604.

[34] B. Wolf et al., First radioactive ions charge bred in REXEBIS at the REX-ISOLDE accelerator, Nucl. Instrum. Meth. B 204 (2003) 428.

[35] F. Wenander, P. Delahaye, R. Scrivens, R. Savreux, The REX-ISOLDE charge breeder as an operational machine, Rev. Sci. Instrum. 77 (2006) 03B104-1-5.

[36] A. Müller and E. Salzborn, Scaling of cross sections for multiple electron transfer to highly charged ions colliding with atoms and molecules, Phys. Lett. A 62 (1977) 391.

[37] REX-ISOLDE collaboration, Radioactive Beam EXperiment at ISOLDE, http://isolde.web.cern.ch/ISOLDE/REX-ISOLDE/index.html.

[38] D. Voulot et al., Radioactive beams at REX-ISOLDE: Present status and latest developments, Nucl. Instrum. Meth. B 266 (2008) 4103.

[39] F. Ames et al., Space-charge effect with REXTRAP, Hyperfine Interactions 132 (2001) 469.

[40] I. Poderea-Aliseda, New developments on preparation of cooled and bunched Radioactive Ion beams at ISOL facilities: the ISCOOL project and the rotating wall cooling, CERN-THESIS-2006-034, Barcelona (2006). 
[41] M. Kowalska et al., Access to the Yield information, https://oraweb.cern.ch/pls/isolde/query tgt.

[42] F. Wenander, An EBIS as heavy ion source for the LHC pre-injector, AIP Conf. Proc. 572 (2001) 48.

[43] R. Becker, O. Brinzanescu and Th. Stöhlker, Limitation of EBIS/T ion yield by radiative recombination, AIP Conf. Proc. 572 (2001) 119.

[44] R. Becker, O. Kester, and Th. Stoehlker, Simulation of charge breeding for trapped ions, J. Phys.: Conf. Ser. 58 (2007) 443.

[45] O. Kester et al., Injection and cooling of HCI's, EURONS charge breeding JRA03, deliverable report D-J03-1.1 and 3.1, http://www.gsi.de/informationen/jofu/EURONS/Deliverables.html.

[46] A. Lapierre et al., The TITAN EBIT charge breeder for mass measurements on highly charged short-lived isotopes - first online operation, submitted to Nucl. Instrum. Meth. A.

[47] R. Kirchner, Review of ISOL target-ion-source systems, Nucl. Instrum. Meth. B 204 (2003) 179.

[48] H. Frånberg, Production of exotic, short lived carbon isotopes in ISOL-type facilities, Doctoral thesis, Bern University, 2008.

[49] P. Delahaye, F. Ames, I. Podadera, R. Savreux and F. Wenander, Recent developments of the radioactive beam preparation at REX-ISOLDE, Eur. Phys. J. A 25 (2005) 739.

[50] M. Marie-Jeane et al., Beam purification, EURONS charge breeding JRA03, deliverable report D-J03-5.1, http://www.gsi.de/informationen/jofu/EURONS/Deliverables.html.

[51] S. Sturm, Implementation of a high resolution mass resolving technique for REXTRAP and investigation of space charge related phenomena, Master Thesis, Universitaet Heidelberg (2007).

[52] A. Gustafsson et al., Mass-selective operation with REXTRAP, submitted to Nucl. Instrum. Meth. A.

[53] V.N. Fedoseyev et al. The ISOLDE laser ion source for exotic nuclei, Hyperfine Interactions 127 (2000) 409.

[54] H. Frånberg et al., Off-line commissioning of the ISOLDE cooler, Nucl. Instrum. Meth. B 266 (2008) 4502.

[55] A. Herlert et al., Mass spectrometry of atomic ions produced by in-trap decay of short-lived nuclides, New J. Phys. 7 (2005) 44.

[56] J. Van de Walle et al., In-trap decay of ${ }^{61} \mathrm{Mn}$ and Coulomb excitation of ${ }^{61} \mathrm{Mn} /{ }^{61} \mathrm{Fe}$, Eur. Phys. J. A 42 (2009) 401.

[57] A. Gustafsson, Investigation of $\beta$-decay induced ion-recoil effects in REX-ISOLDE and mass-separation methods with REXTRAP, Diploma Thesis, Fachhochschule Wiesbaden (2009).

[58] E. Beebe et al., TEST EBIS operation and component development for the RHIC EBIS, J. Phys. Conf. Ser. 2 (2004) 164.

[59] A. Pikin et al., RHIC EBIS: basics of design and status of commissioning, in International Symposium on Electron Beam Ion Sources and Traps, April $7^{\text {th }}-10^{\text {th }} 2010$, Stockholm, Sweden.

[60] O. Kester et al., The MSU/NSCL re-accelerator ReA3, Proc. SRF2009, Berlin, Germany.

[61] S. Schwarz et al., An electron beam ion trap for the NSCL reaccelerator, Nucl. Instrum. Meth. B 266 (2008) 4466.

[62] S. Schwarz et al., EBIS/T charge breeders for intense rare isotope beams, in International Symposium on Electron Beam Ion Sources and Traps, April $7^{\text {th }}-10^{\text {th }} 2010$, Stockholm, Sweden.

[63] S. Schwarz et al., A high-current electron beam ion trap as a charge breeder for the reacceleration of rare isotopes at the NSCL, Rev. Sci. Instrum. 79 (2008) 02A706. 
[64] E. Gavartin, Acceptance calculations for a charge breeder based on an electron beam ion trap, MSc. Thesis, Michigan State University, 2008.

[65] G. Savard et al., Radioactive beams from gas catchers: The CARIBU facility, Nucl. Instrum. Meth. B 266 (2008) 4086.

[66] P. Ostroumov et al., EBIS - Charge Breeder for CARIBU: choice of strategy and initial studies, in International Symposium on Electron Beam Ion Sources and Traps, April $7^{\text {th }}-10^{\text {th }} 2010$, Stockholm, Sweden.

[67] M. Froese, The TITAN Electron Beam Ion Trap: assembly, characterization and first tests, MSc. Thesis, University of Manitoba, Canada (2006).

[68] J. Dilling, P. Bricault, M. Smith, H-J. Kluge and TITAN collaboration, The proposed TITAN facility at ISAC for very precise mass measurements on highly charged short-lived isotopes, Nucl. Instrum. Meth. B 204 (2003) 492.

[69] V. Variale et al., Selective containment in EBIS for charge breeding applications, Nucl. Instrum. Meth. A 592 (2008) 9.

[70] A. Lapierre et al., TITAN project: Towards mass measurements in HCI, in International Symposium on Electron Beam Ion Sources and Traps, April $7^{\text {th }}-10^{\text {th }} 2010$, Stockholm, Sweden.

[71] F. Herfurth et al., Precision measurements with highly charged ions at rest: The HITRAP project at GSI, Int. J. Mass Spectrom. 251 (2006) 266.

[72] B. O'Rourke et al., The SPARC EBIT at GSI; commissioning and future plans at the HITRAP beamline, J. Phys. Conf. Ser. 164 (2009) 012103.

[73] A. Thorn et al., Charge breeding with the SPARC-EBIT, annual report GSI, 2009.

[74] A. Sokolov et al., Successful charge breeding in the SPARC EBIT, in International Symposium on Electron Beam Ion Sources and Traps, April $7^{\text {th }}-10^{\text {th }} 2010$, Stockholm, Sweden.

[75] D. Rodriguez et al., MATS and LaSpec Technical Design Report, Eur. Phys. J. Special Topics 183 (2010) 1.

[76] F. Gerigk and M. Vretenar eds., Linac4 Technical Design Report, CERN-AB-2006-084 ABP/RF (2006).

[77] R.C. York et al., FRIB: a new accelerator facility for the production of rare isotope beams, in proceedings of SRF2009, Berlin, Germany (2009).

[78] J. Cornell ed., EURISOL Design Study Final Report, http://www.eurisol.org/eurisol_design_study_final_report-1156.html (2009).

[79] P. Delahaye O. Kester, C. Barton, T. Lamy, M. Marie-Jeanne and F. Wenander, Evaluation of charge-breeding options for EURISOL, submitted to Eur. Phys. J. A.

[80] S. Koscielniak, An electron linac photo-fission driver for the rare isotope program at TRIUMF, Proc. SRF2009, Berlin, Germany.

[81] T. Thuillier, T. Lamy, L. Latrasse and J. Angot, First Plasma of the A-PHOENIX electron cyclotron resonance ion source, Rev. Sci. Instrum. 79 (2008) 02A330. 